\title{
Multiphase-Linear Ranking Functions and their Relation to Recurrent Sets
}

\author{
Amir M. Ben-Amram¹, Jesús J. Doménech ${ }^{\dagger 2}$, and Samir Genaim ${ }^{\ddagger 2}$ \\ ${ }^{1}$ The Academic College of Tel-Aviv Yaffo \\ ${ }^{2}$ Complutense University of Madrid
}

January 15, 2019

\begin{abstract}
Multiphase ranking functions (M $\Phi$ Rs) are tuples $\left\langle f_{1}, \ldots, f_{d}\right\rangle$ of linear functions that are often used to prove termination of loops in which the computation progresses through a number of "phases". Our work provides new insights regarding such functions for loops described by a conjunction of linear constraints (Single-Path Constraint loops). The decision problem existence of a $M \Phi R F$ asks to determine whether a given $S L C$ loop admits a M $\Phi R F$; and the corresponding bounded decision problem restricts the search to M $\Phi R F$ s of depth $d$, where the parameter $d$ is part of the input. The algorithmic and complexity aspects of the bounded problem have been completely settled in a recent work. In this paper we make progress regarding the existence problem, without a given depth bound. In particular, we present an approach that reveals some important insights into the structure of these functions. Interestingly, it relates the problem of seeking $M \Phi R F$ s to that of seeking recurrent sets (used to prove non-termination). It also helps in identifying classes of loops for which M $\Phi R F$ s are sufficient. Our research has led to a new representation for single-path loops, the difference polyhedron replacing the customary transition polyhedron. This representation yields new insights on M $\Phi R F$ s and SLC loops in general. For example, a result on bounded $S L C$ loops becomes straightforward.
\end{abstract}

*amirben@mta.ac.il

†jdomenec@ucm.es

${ }^{\ddagger}$ samir.genaim@fdi.ucm.es 


\section{Introduction}

Proving that a program will not go into an infinite loop is one of the most fundamental tasks of program verification, and has been the subject of voluminous research. Perhaps the best known, and often used, technique for proving termination is that of ranking functions. This consists of finding a function that maps program states into the elements of a well-founded ordered set, such that it decreases when applied to consecutive states. This implies termination since infinite descent is impossible in a well-founded order.

Unlike termination of programs in general, which is undecidable, the algorithmic problems of detection (deciding the existence) or generation (synthesis) of a ranking function can well be solvable, given certain choices of the program representation, and the class of ranking function. There is a considerable amount of research in this direction, in which different kinds of ranking functions for different kinds of program representations were considered. In some cases the algorithmic problems have been completely settled, and efficient algorithms provided, while other cases remain open. Besides proving termination, some classes of ranking functions also serve to bound the length of the computation (an iteration bound), useful in applications such as cost analysis and loop optimization [21, 3, 2, 13].

We focus on single-path linear-constraint loops ( $S L C$ loops), where a state is described by the values of a finite set of numerical variables, and the effect of a transition (one iteration of the loop) is described by a conjunction of linear constraints. We consider the setting of integer-valued variables, as well as rational-valued (or real-valued) variables. Here is an example of this loop representation (formally defined in Section 2); primed variables $x^{\prime}, y^{\prime}, \ldots$ refer to the state following the transition.

$$
\text { while }\left(x_{1} \geq-x_{3}\right) \text { do } x_{1}^{\prime}=x_{1}+x_{2}, x_{2}^{\prime}=x_{2}+x_{3}, x_{3}^{\prime}=x_{3}-1
$$

Note that by $x_{1}^{\prime}=x_{1}+x_{2}$ we mean an equation, not an assignment statement. The description of a loop might involve linear inequalities rather than equations, and consequently be nondeterministic. It is a standard procedure to compile sequential code (or approximate it) into such representation using various techniques. We assume the "constraint loop" to be given, and do not concern ourselves with the orthogonal topic of extracting such loops from general programs. This constraint representation may be extended to represent branching in the loop body, a so-called multiple-path loop; in the current work we do not consider such loops. However, SLC loops are important, e.g., in approaches that reduce a question about a whole program to questions about simple loops [23, 28, 20, 18, 19]; see [30] for references that show the importance of such loops in other fields.

Several types of ranking functions have been suggested for $S L C$ loops; linear ranking functions $(L R F s)$ are probably the most known. In this case, we seek a func- 
tion $\rho\left(x_{1}, \ldots, x_{n}\right)=a_{1} x_{1}+\cdots+a_{n} x_{n}+a_{0}$ such that (i) $\rho(\mathbf{x}) \geq 0$ for any valuation $\mathbf{x}=\left\langle x_{1}, \ldots, x_{n}\right\rangle$ that satisfies the loop constraints (i.e., an enabled state); and (ii) $\rho(\mathbf{x})-\rho\left(\mathbf{x}^{\prime}\right) \geq 1$ for any transition leading from $\mathbf{x}$ to $\mathbf{x}^{\prime}=\left\langle x_{1}^{\prime}, \ldots, x_{n}^{\prime}\right\rangle$. The algorithmic problems of existence and synthesis of LRFs has been completely settled [21, 34, 16, 32, 7], for both integer-valued and rational-valued variables, not only for $S L C$ loops but rather for control-flow graphs.

$L R F s$ do not suffice for all terminating $S L C$ loops, e.g., Loop (1) does not have a $L R F$, and in such case, one may resort to an argument that combines several linear functions to capture a more complex behavior. A common such argument is one that uses lexicographic ranking functions, where a tuple of linear functions is required to decreases lexicographically when moving from one state to another. In this paper we are interested in a special case of the lexicographic order argument that is called Multiphase ranking functions ( $\mathrm{M} \Phi R \mathrm{R}$ for short). Intuitively, a $\mathrm{M} \Phi \mathrm{RF}$ is a tuple $\left\langle f_{1}, \ldots, f_{d}\right\rangle$ of linear functions that define phases of the loop that are linearly ranked, as follows: $f_{1}$ decreases on all transitions, and when it becomes negative $f_{2}$ starts to decrease, and when $f_{2}$ becomes negative, $f_{3}$ starts to decrease, etc. For example, Loop (11) has the $\operatorname{M} \Phi R F\left\langle x_{3}+1, x_{2}+1, x_{1}\right\rangle$. The general definition (see Section 2) allows for an arbitrary number $d$ of linear components; we refer to $d$ as depth, intuitively the number of phases.

The decision problem Existence of a $M \Phi R F$ asks to determine whether a given $S L C$ loop admits a M $\Phi R F$. The bounded decision problem restricts the search to M $\Phi$ RFs of depth $d$, where the parameter $d$ is part of the input. The complexity and algorithmic aspects of the bounded version of the $M \Phi R F$ problem has been completely settled in [8]. The decision problem is PTIME for $S L C$ loops with rational-valued variables, and coNPcomplete for $S L C$ loops with integer-valued variables; synthesizing M $\Phi$ RFs, when they exist, can be performed in polynomial and exponential time, respectively. In addition, [8] shows that for $S L C$ loops M $\Phi R F$ s has the same power as general lexicographic orders, and that, surprisingly, M $\Phi$ Rs induce a linear bound on the number of iterations of $S L C$ loops. The problem of deciding whether a given $S L C$ admits a M $\Phi R F$, without a given bound on the depth, is still open.

In practice, termination analysis tools search for M $M R F$ s starting by depth 1 and incrementally increase the depth until they find one, or reach a predefined limit, after which the returned answer is don't know. Clearly, finding a theoretical upper-bound on the depth of a $M \Phi R F$, given the loop, would also settle this problem. As shown in [8], such bound must depend not only on the number of constraints or variables, but rather on the coefficients used in the constraints.

In this paper we make progress towards solving the problem of existence of a $M \Phi R F$, i.e., seeking a $M \Phi R F$ without a given bound on the depth. In particular, we present an algorithm for seeking M $\Phi R F$ s that reveals important insights on the structure of these 
ranking functions. In a nutshell, the algorithm stars from the set of transitions of the given $S L C$ loop, which is a polyhedron, and iteratively removes transitions $\left(\mathbf{x}, \mathbf{x}^{\prime}\right)$ such that $\rho(\mathbf{x})-\rho\left(\mathbf{x}^{\prime}\right)>0$ for some function $\rho(\mathbf{x})=\vec{a} \cdot \mathbf{x}+b$ that is non-negative on all enabled states. The process continues iteratively, since after removing some transitions, more functions $\rho$ may satisfy the non-negativity condition, and they may eliminate additional transitions in the next iteration. When all transitions are eliminated in a finite number of iterations, we can construct a M MRF using the $\rho$ functions; and when reaching a situation in which no transition can be eliminated, we prove that we have actually reached a recurrent set that witnesses nontermination.

The algorithm always finds a M $\Phi R F$ if one exists, and in many cases it finds a recurrent set (see the discussion on experiments in Section 6) when the loop is not terminating, however, it is not a decision procedure as it diverges in some cases. Nonetheless, our algorithm provides important insights on the structure of M $\Phi$ RFs. Apart from revealing a relation between seeking $M \Phi R F$ s and seeking recurrent sets of a particular form, these insights are also useful for finding classes of $S L C$ loops for which, when terminating, there is always a M $\Phi \mathrm{RF}$. We shall prove this for two classes of $S L C$ loops that have been considered before in previous work.

Our research, in addition, has led to a new representation for $S L C$ loops, that we refer to as the displacement representation, that provides us with new tools for studying termination of $S L C$ loops in general, and existence of a $M \Phi R F$ in particular. In this representation a transition $\left(\mathbf{x}, \mathbf{x}^{\prime}\right)$ is represented as $(\mathbf{x}, \mathbf{y})$ where $\mathbf{y}=\mathbf{x}^{\prime}-\mathbf{x}$. We show that using this representation our algorithm can be formalized in a simple way that avoids computing the $\rho$ functions mentioned above (which might be expensive), and such that the existence of a M $\Phi R F$ is then equivalent to checking for unsatisfiability of a linear constraint system. As an evidence on the usefulness of this representation in general, we show that some nontrivial observations on termination of bounded $S L C$ loops are made straightforward in this representation, while they are not easy to see in the normal representation.

The article is organized as follows. Section 2 gives precise definitions and necessary background. Section 3 describes our algorithm and its possible outcomes. Section 4 discusses classes of $S L C$ loops for which, when terminating, there is always a M $\Phi$ RF. Section 5 discusses the displacement representation for $S L C$ loops. Finally, in Section 6 we conclude and discuss some related work.

\section{Preliminaries}

In this section we give the fundamental definitions for this paper: recall some definitions regarding (integer) polyhedra and mention some important properties of these 
definitions, we define the class of loops we study, the type of ranking functions, and the notion of recurrent sets.

\subsection{Polyhedra}

A rational convex polyhedron $\mathcal{P} \subseteq \mathbb{Q}^{n}$ (polyhedron for short) is the set of solutions of a set of inequalities $A \mathbf{x} \leq \mathbf{b}$, namely $\mathcal{P}=\left\{\mathbf{x} \in \mathbb{Q}^{n} \mid A \mathbf{x} \leq \mathbf{b}\right\}$, where $A \in \mathbb{Q}^{m \times n}$ is a rational matrix of $n$ columns and $m$ rows, $\mathbf{x} \in \mathbb{Q}^{n}$ and $\mathbf{b} \in \mathbb{Q}^{m}$ are column vectors of $n$ and $m$ rational values respectively. We say that $\mathcal{P}$ is specified by $A \mathbf{x} \leq \mathbf{b}$. If $\mathbf{b}=\mathbf{0}$, then $\mathcal{P}$ is a cone. The set of recession directions of a polyhedron $\mathcal{P}$ specified by $A \mathbf{x} \leq \mathbf{b}$, also known as its recession cone, is the set rec.cone $(\mathcal{P})=\left\{\mathbf{y} \in \mathbb{Q}^{n} \mid A \mathbf{y} \leq \mathbf{0}\right\}$.

For a given polyhedron $\mathcal{P} \subseteq \mathbb{Q}^{n}$ we let $I(\mathcal{P})$ be $\mathcal{P} \cap \mathbb{Z}^{n}$, i.e., the set of integer points of $\mathcal{P}$. The integer hull of $\mathcal{P}$, commonly denoted by $\mathcal{P}_{I}$, is defined as the convex hull of $I(\mathcal{P})$, i.e., every rational point of $\mathcal{P}_{I}$ is a convex combination of integer points. It is known that $\mathcal{P}_{I}$ is also a polyhedron, and that rec.cone $(\mathcal{P})=$ rec.cone $\left(\mathcal{P}_{I}\right)$. An integer polyhedron is a polyhedron $\mathcal{P}$ such that $\mathcal{P}=\mathcal{P}_{I}$. We also say that such an integer polyhedron is integral.

Polyhedra also have a generator representation in terms of vertices and rays, written as $\mathcal{P}=$ conv.hull $\left\{\mathbf{x}_{1}, \ldots, \mathbf{x}_{m}\right\}+$ cone $\left\{\mathbf{y}_{1}, \ldots, \mathbf{y}_{t}\right\}$. This means that $\mathbf{x} \in \mathcal{P}$ iff $\mathbf{x}=$ $\sum_{i=1}^{m} a_{i} \cdot \mathbf{x}_{i}+\sum_{j=1}^{t} b_{j} \cdot \mathbf{y}_{j}$ for some rationals $a_{i}, b_{j} \geq 0$, where $\sum_{i=1}^{m} a_{i}=1$. Note that $\mathbf{y}_{1}, \ldots, \mathbf{y}_{t}$ are the recession directions of $\mathcal{P}$, i.e., $\mathbf{y} \in \operatorname{rec} . \operatorname{cone}(\mathcal{P})$ iff $\mathbf{y}=\sum_{j=1}^{t} b_{j} \cdot \mathbf{y}_{j}$ for some rationals $b_{j} \geq 0$. If $\mathcal{P}$ is integral, then there is a generator representation in which all $\mathbf{x}_{i}$ and $\mathbf{y}_{j}$ are integers.

Let $\mathcal{P} \subseteq \mathbb{Q}^{n+m}$ be a polyhedron, and let $\left(\begin{array}{l}\mathbf{x} \\ \mathbf{y}\end{array}\right) \in \mathcal{P}$ be such that $\mathbf{x} \in \mathbb{Q}^{n}$ and $\mathbf{y} \in \mathbb{Q}^{m}$. The projection of $\mathcal{P}$ onto the $\mathbf{x}$-space is defined as $\operatorname{proj}_{\mathbf{x}}(\mathcal{P})=\left\{\mathbf{x} \in \mathbb{Q}^{n} \mid \exists \mathbf{y} \in\right.$ $\mathbb{Q}^{m}$ such that $\left.\left(\begin{array}{l}\mathbf{x} \\ \mathbf{y}\end{array}\right) \in \mathcal{P}\right\}$. We will need the following lemmas later.

LEMMA 2.1. $\operatorname{proj}_{\mathbf{x}}(\operatorname{rec} \cdot \operatorname{cone}(\mathcal{P}))=\operatorname{rec} \cdot \operatorname{cone}\left(\operatorname{proj}_{\mathbf{x}}(\mathcal{P})\right)$.

Proof. A polyhedron $\mathcal{P}$ whose variables are split into two sets, $\mathbf{x}$ and $\mathbf{y}$, can be represented in the form $A \mathbf{x}+G \mathbf{y} \leq \mathbf{b}$ for matrices $A, G$ and a vector $\mathbf{b}$ of matching dimensions. Then [17, Theorem 11.11] states that $\operatorname{proj}_{\mathbf{x}}(\mathcal{P})$ is specified by the constraints $V(\mathbf{b}-A \mathbf{x}) \geq \mathbf{0}$ for a certain matrix $V$ determined by $G$ only. From this it follows that rec.cone $\left(\operatorname{proj}_{\mathbf{x}}(\mathcal{P})\right)=\{\mathbf{x}: V A \mathbf{x} \leq \mathbf{0}\}$. But we can also apply the theorem to rec.cone $(\mathcal{P})$, which is specified by $A \mathbf{x}+G \mathbf{y} \leq \mathbf{0}$, and we get the same result $\operatorname{proj}_{\mathbf{x}}(\operatorname{rec} . \operatorname{cone}(\mathcal{P}))=\{\mathbf{x}: V A \mathbf{x} \leq \mathbf{0}\}$.

LEMMA 2.2. Given a polyhedron $\mathcal{P} \neq \emptyset$, and linear functions $\rho_{1}, \ldots, \rho_{k}$ such that

(i) $\mathbf{x} \in \mathcal{P} \rightarrow \rho_{1}(\mathbf{x})>0 \vee \cdots \vee \rho_{k-1}(\mathbf{x})>0 \vee \rho_{k}(\mathbf{x}) \geq 0$ 
(ii) $\mathbf{x} \in \mathcal{P} \not \rightarrow \rho_{1}(\mathbf{x})>0 \vee \cdots \vee \rho_{k-1}(\mathbf{x})>0$

There exist non-negative constants $\mu_{1}, \ldots, \mu_{k-1}$ such that $\mathbf{x} \in \mathcal{P} \rightarrow \mu_{1} \rho_{1}(\mathbf{x})+\cdots+$ $\mu_{k-1} \rho_{k-1}(\mathbf{x})+\rho_{k}(\mathbf{x}) \geq 0$.

Proof. Let $\mathcal{P}$ be $B \mathbf{x} \leq \mathbf{c}, \rho_{i}=\vec{a}_{i} \cdot \mathbf{x}-b_{i}$, then $(i)$ is equivalent to infeasibility of

$$
B \mathbf{x} \leq \mathbf{c} \wedge A \mathbf{x} \leq \mathbf{b} \wedge \vec{a}_{k} \cdot \mathbf{x}<b_{k}
$$

where $A$ consists of the $k-1$ rows $\vec{a}_{i}$, and $\mathbf{b}$ of corresponding $b_{i}$. However, $B \mathbf{x} \leq$ $\mathbf{c} \wedge A \mathbf{x} \leq \mathbf{b}$ is assumed to be feasible.

According to Motzkin's transposition theorem [33, Corollary 7.1k, Page 94], this

implies that there are row vectors $\vec{\lambda}, \vec{\lambda}^{\prime} \geq 0$ and a constant $\mu \geq 0$ such that the following is true:

$$
\vec{\lambda} B+\vec{\lambda}^{\prime} A+\mu a_{k}=0 \wedge \vec{\lambda} \mathbf{c}+\vec{\lambda}^{\prime} \mathbf{b}+\mu b_{k} \leq 0 \wedge\left(\mu \neq 0 \vee \vec{\lambda} \mathbf{c}+\vec{\lambda}^{\prime} \mathbf{b}+\mu b_{k}<0\right)
$$

Now, if (3) is true, then for all $\mathrm{x} \in \mathcal{P}$,

$$
\begin{aligned}
\left(\sum_{i} \lambda_{i}^{\prime} \rho_{i}(\mathbf{x})\right)+\mu \rho_{k}(\mathbf{x}) & =\vec{\lambda}^{\prime} A \mathbf{x}-\vec{\lambda}^{\prime} \mathbf{b}+\mu a_{k} \mathbf{x}-\mu b_{k} \\
& =-\vec{\lambda} B \mathbf{x}-\vec{\lambda}^{\prime} \mathbf{b}-\mu b_{k} \geq-\vec{\lambda} \mathbf{c}-\vec{\lambda}^{\prime} \mathbf{b}-\mu b_{k} \geq 0
\end{aligned}
$$

where if $\mu=0$, the last inequality must be strict. However, if $\mu=0$, then $\vec{\lambda} B+\vec{\lambda}^{\prime} A=0$, so by feasibility of $B \mathbf{x} \leq \mathbf{c}$ and $A \mathbf{x} \leq \mathbf{b}$, this implies $\vec{\lambda} \mathbf{c}+\vec{\lambda}^{\prime} \mathbf{b} \geq 0$, a contradiction. Thus, $\left(\sum_{i} \lambda_{i}^{\prime} \rho_{i}\right)+\mu \rho_{k} \geq 0$ on $\mathcal{P}$ and $\mu>0$. Dividing by $\mu$ we obtain the conclusion of the lemma.

\subsection{Single-Path Linear-Constraint Loops}

A single-path linear-constraint loop ( $S L C$ loop) over $n$ variables $x_{1}, \ldots, x_{n}$ has the form

$$
\text { while }(B \mathbf{x} \leq \mathbf{b}) \text { do } A \mathbf{x}+A^{\prime} \mathbf{x}^{\prime} \leq \mathbf{c}
$$

where $\mathbf{x}=\left(x_{1}, \ldots, x_{n}\right)^{\mathrm{T}}$ and $\mathbf{x}^{\prime}=\left(x_{1}^{\prime}, \ldots, x_{n}^{\prime}\right)^{\mathrm{T}}$ are column vectors, and for some $p, q>0, B \in \mathbb{Q}^{p \times n}, A, A^{\prime} \in \mathbb{Q}^{q \times n}, \mathbf{b} \in \mathbb{Q}^{p}, \mathbf{c} \in \mathbb{Q}^{q}$. The constraint $B \mathbf{x} \leq \mathbf{b}$ is called the loop condition (a.k.a. the loop guard) and the other constraint is called the update. The update is called deterministic if, for a given $\mathbf{x}$ (satisfying the loop condition) there is at most one $\mathbf{x}^{\prime}$ satisfying the update constraint. The update is called affine linear if it can be rewritten as

$$
\mathbf{x}^{\prime}=U \mathbf{x}+\mathbf{c}
$$


for a matrix $U \in \mathbb{Q}^{n \times n}$ and vector $\mathbf{c} \in \mathbb{Q}^{n}$. We say that the loop is a rational loop if $\mathbf{x}$ and $\mathbf{x}^{\prime}$ range over $\mathbb{Q}^{n}$, and that it is an integer loop if they range over $\mathbb{Z}^{n}$. One could also allow variables to take any real-number values, but for the problems we study, where the constraints are expressed by rational numbers, this very rarely differs from the rational case (when it does, we comment on that explicitly).

We say that there is a transition from a state $\mathbf{x} \in \mathbb{Q}^{n}$ to a state $\mathbf{x}^{\prime} \in \mathbb{Q}^{n}$, if $\mathbf{x}$ satisfies the loop condition and $\mathbf{x}$ and $\mathbf{x}^{\prime}$ satisfy the update constraint. A transition can be seen as a point $\left(\begin{array}{l}\mathbf{x} \\ \mathbf{x}^{\prime}\end{array}\right) \in \mathbb{Q}^{2 n}$, where its first $n$ components correspond to $\mathbf{x}$ and its last $n$ components to $\mathbf{x}^{\prime}$. For ease of notation, we denote $\left(\begin{array}{l}\mathbf{x} \\ \mathbf{x}^{\prime}\end{array}\right)$ by $\mathbf{x}^{\prime \prime}$. The set of all transitions $\mathbf{x}^{\prime \prime} \in \mathbb{Q}^{2 n}$, of a given $S L C$ loop, will be denoted by $\mathcal{Q}$ and is specified by the set of inequalities $A^{\prime \prime} \mathbf{x}^{\prime \prime} \leq \mathbf{c}^{\prime \prime}$ where

$$
A^{\prime \prime}=\left(\begin{array}{cc}
B & 0 \\
A & A^{\prime}
\end{array}\right) \quad \mathbf{c}^{\prime \prime}=\left(\begin{array}{l}
\mathbf{b} \\
\mathbf{c}
\end{array}\right)
$$

and $B, A, A^{\prime}$, $\mathbf{c}$ and $\mathbf{b}$ are those of (44). We call $\mathcal{Q}$ the transition polyhedron. For the purpose of this article, the loop is fully represented by this polyhedron (in examples, we may use a more readable form as (4) ). For integer loops, the set of transitions is denoted by $I(\mathcal{Q})$.

\subsection{Multi-Phase Ranking Functions}

An affine linear function $\rho: \mathbb{Q}^{n} \rightarrow \mathbb{Q}$ is a function of the form $\rho(\mathbf{x})=\vec{a} \cdot \mathbf{x}+b$ where $\vec{a} \in \mathbb{Q}^{n}$ is a row vector and $b \in \mathbb{Q}$. For a given function $\rho$, we define the function $\Delta \rho: \mathbb{Q}^{2 n} \mapsto \mathbb{Q}$ as $\Delta \rho\left(\mathbf{x}^{\prime \prime}\right)=\rho(\mathbf{x})-\rho\left(\mathbf{x}^{\prime}\right)$.

Definition 2.3 (M $(\mathrm{RF})$. Given a set of transitions $T \subseteq \mathbb{Q}^{2 n}$, we say that $\tau=$ $\left\langle\rho_{1}, \ldots, \rho_{d}\right\rangle$ is a M $\operatorname{RRF}$ (of depth $d$ ) for $T$ if for every $\mathbf{x}^{\prime \prime} \in T$ there is an index $i$ such that:

$$
\begin{aligned}
\forall j \leq i . \Delta \rho_{j}\left(\mathbf{x}^{\prime \prime}\right) & \geq 1, \\
\rho_{i}(\mathbf{x}) & \geq 0, \\
\forall j<i . & \rho_{j}(\mathbf{x}) \leq 0 .
\end{aligned}
$$

We say that $\mathbf{x}^{\prime \prime}$ is ranked by $\rho_{i}$ (for the minimal such $i$ ).

It is not hard to see that a $\mathrm{M} \Phi \mathrm{RF}\left\langle\rho_{1}\right\rangle$ of depth $d=1$ is a linear ranking function $(L R F)$. On the other hand, if the M $\Phi R F$ is of depth $d>1$, it implies that if $\rho_{1}(\mathbf{x}) \geq 0$, transition $\mathbf{x}^{\prime \prime}$ is ranked by $\rho_{1}$, while if $\rho_{1}(\mathbf{x})<0,\left\langle\rho_{2}, \ldots, \rho_{d}\right\rangle$ becomes a M $\Phi R F$. This agrees with the intuitive notion of "phases." We further note that, for loops specified 
by polyhedra (namely, $S L C$ loops as in (4)), making the inequality (8) strict results in the same class of ranking functions (we chose the definition that is easier to work with), and, similarly, we can replace (6) by $\Delta \rho_{j}\left(\mathbf{x}^{\prime \prime}\right)>0$, obtaining an equivalent definition (up to multiplication of the $\rho_{i}$ by some constants). We say that $\tau$ is irredundant if removing any component invalidates the $M \Phi R F$. Finally, it is convenient to allow an empty tuple as a M $\Phi \mathrm{RF}$, of depth 0 , for the empty set.

The decision problem Existence of a $M \Phi R F$ asks to determine whether a given $S L C$ loop admits a M $\Phi R F$. The bounded decision problem restricts the search to M $\Phi$ RFs of depth at most $d$, where the parameter $d$ is part of the input.

\subsection{Recurrent Sets}

A recurrent set is a set of states that witnesses nontermination of a given $S L C$ loop $\mathcal{Q}$. It is commonly defined as a set of states $S \subseteq \operatorname{proj}_{\mathbf{x}}(\mathcal{Q})$ where for any $\mathbf{x} \in S$ there is $\mathbf{x}^{\prime} \in S$ such that $\left(\mathbf{x}, \mathbf{x}^{\prime}\right) \in \mathcal{Q}$. This clearly proves the existence of an infinite run. In this article we use a slightly different notion that uses transitions instead of states.

Definition 2.4. Give a $S L C$ loop $\mathcal{Q}$, we say that $S \subseteq \mathcal{Q}$ is a recurrent set of transitions if $\operatorname{proj}_{\mathbf{x}^{\prime}}(S) \subseteq \operatorname{proj}_{\mathbf{x}}(S)$.

Note that $\operatorname{proj}_{\mathbf{x}}(S)$ is a recurrent set of states as explained above. It is known that the largest recurrent set (both for states and transitions) of a given $S L C$ loop is convex. In the rest of this article we will mostly be interested in the case in which $S$ is a closed polyhedron.

\section{An algorithm for inferring $\mathrm{M} \Phi \mathbf{R F}$}

In this section we describe our algorithm for deciding the existence of (and constructing) M $\Phi$ RFs, which is also able to find recurrent sets for certain nonterminating $S L C$ loops. In what follows we assume a given $S L C$ loop $\mathcal{Q}$ where variables range over the rationals (or reals), the case of integer variables is always discussed after considering the rational case.

Let us start with an intuitive description of the algorithm and its possible outcomes. Our work started with the following crucial observation: given linear functions $\rho_{1}, \ldots, \rho_{l}$ such that

- $\rho_{1}, \ldots, \rho_{l}$ are nonnegative over $\operatorname{proj}_{\mathbf{x}}(\mathcal{Q})$, i.e., over all enabled states;

- for some $\rho_{i}$, we have $\Delta \rho_{i}\left(\mathbf{x}^{\prime \prime}\right)>0$ for at least one transition $\mathbf{x}^{\prime \prime} \in \mathcal{Q}$; and 
- $\mathcal{Q}^{\prime}=\mathcal{Q} \wedge \Delta \rho_{1}\left(\mathbf{x}^{\prime \prime}\right) \leq 0 \wedge \cdots \wedge \Delta \rho_{l}\left(\mathbf{x}^{\prime \prime}\right) \leq 0$ has a M $\Phi R F$ of depth $d$

then $\mathcal{Q}$ has a $M \Phi R F$ of depth at most $d+1$. The proof of this lemma is constructive, i.e., given a $\mathrm{M} \Phi \mathrm{RF} \tau^{\prime}$ for $\mathcal{Q}^{\prime}$, we can construct a $\operatorname{M} \Phi \mathrm{RF} \tau$ for $\mathcal{Q}$ using conic combinations of the components of $\tau^{\prime}$ and $\rho_{1}, \ldots, \rho_{l}$.

Let us assume that we have a procedure $F(\mathcal{Q})$ that picks some candidate functions $\rho_{1}, \ldots, \rho_{l}$, i.e., nonnegative over $\operatorname{proj}_{\mathbf{x}}(\mathcal{Q})$, and computes $F(\mathcal{Q})=\mathcal{Q} \wedge \Delta \rho_{1}\left(\mathbf{x}^{\prime \prime}\right) \leq$ $0 \wedge \cdots \wedge \Delta \rho_{l}\left(\mathbf{x}^{\prime \prime}\right) \leq 0$. Clearly, if $F^{d}(\mathcal{Q})=\emptyset$, for some $d>0$, then using the above observation we can conclude that $\mathcal{Q}$ has a M $\Phi R F$ of depth at most $d$. Obviously, the difficult part in defining $F$ is how to find functions $\rho_{1}, \ldots, \rho_{l}$, and, moreover, how to choose ones to find the optimal depth $d$, equivalently, so that if $\mathcal{Q}$ has a M $\Phi R F$ of optimal depth $d$ then $F^{d}(\mathcal{Q})=\emptyset$. For this, we note that the set of all nonnegative functions over $\operatorname{proj}_{\mathbf{x}}(\mathcal{Q})$ is a polyhedral cone, and thus it has generators $\rho_{1}, \ldots, \rho_{l}$ that can be effectively computed. These $\rho_{1}, \ldots, \rho_{l}$ turn to be the right candidates to use. In addition, when using these candidates, we prove that for cases in which we make no progress, i.e., we get $F^{i-1}(\mathcal{Q})=F^{i}(\mathcal{Q})$, then we have actually reached a recurrent set that witnesses nontermination.

The rest of this section is organized as follow. In Section 3.1 we present the algorithm and discuss how it is used to decide existence of M $\Phi R F$ s; in Section 3.2 we discuss how the algorithm can infer recurrent sets; and in Section 3.3 we discuss cases where the algorithm does not terminate and raise some questions on what happens in the limit.

\subsection{Deciding existence of M MRFs}

We start by formally defining the set of all nonnegative functions over a given polyhedron $\mathcal{S} \subseteq \mathbb{Q}^{n}$.

Definition 3.1. The set of all nonnegative functions over a polyhedron $\mathcal{S} \subseteq \mathbb{Q}^{n}$, is defined as $\mathcal{S}^{\#}=\left\{(\vec{a}, b) \in \mathbb{Q}^{n+1} \mid \forall \mathbf{x} \in \mathcal{S} . \vec{a} \cdot \mathbf{x}+b \geq 0\right\}$.

It is known that $\mathcal{S}^{\#}$ is a polyhedral cone [33, p. 112]. Equivalently, it is generated by a finite set of rays $\left(\vec{a}_{1}, b_{1}\right), \ldots,\left(\vec{a}_{l}, b_{l}\right)$. The cone generated by $\vec{a}_{1}, \ldots, \vec{a}_{l}$ is known as the dual of the cone rec.cone $\left(\operatorname{proj}_{\mathbf{x}}(\mathcal{S})\right)$ - we make use of this in Section 5 , These rays are actually the ones that are important for the algorithm, as can be seen in the definition below, however, in the definition of $\mathcal{S}^{\#}$ we included the $b_{i}$ 's as they makes some statements smoother. Note that $\mathcal{S}$ is equal to the intersection of all half-spaces defined the elements of $\mathcal{S}^{\#}$, i.e., $\mathcal{S}=\wedge\left\{\vec{a} \cdot \mathbf{x}+b \geq 0 \mid(\vec{a}, b) \in \mathcal{S}^{\#}\right\}$, since it is a closed convex set. 
Definition 3.2. Let $\mathcal{Q}$ be a $S L C$ loop, and define

$$
F(\mathcal{Q})=\mathcal{Q} \wedge \vec{a}_{1} \cdot \mathbf{x}-\vec{a}_{1} \cdot \mathbf{x}^{\prime} \leq 0 \wedge \cdots \wedge \vec{a}_{l} \cdot \mathbf{x}-\vec{a}_{l} \cdot \mathbf{x}^{\prime} \leq 0
$$

where $\left(\vec{a}_{1}, b_{1}\right), \ldots,\left(\vec{a}_{l}, b_{l}\right)$ are the generators of $\operatorname{proj}_{\mathbf{x}}(\mathcal{Q})^{\#}$.

Note that each $\vec{a}_{i} \cdot \mathbf{x}-\vec{a}_{i} \cdot \mathbf{x}^{\prime} \leq 0$ above is actually $\Delta \rho_{i}\left(\mathbf{x}^{\prime \prime}\right) \leq 0$ where $\rho_{i}=\vec{a}_{i} \cdot \mathbf{x}+b_{i} \leq 0$. Intuitively, $F(\mathcal{Q})$ removes from $\mathcal{Q}$ all transitions $\mathbf{x}^{\prime \prime}$ for which there is $(\vec{a}, b) \in \operatorname{proj}_{\mathbf{x}}(\mathcal{Q})^{\#}$ such that $\vec{a} \cdot \mathbf{x}-\vec{a} \cdot \mathbf{x}^{\prime}>0$. This is because any $(\vec{a}, b) \in \operatorname{proj}_{\mathbf{x}}(\mathcal{Q})^{\#}$ is a conic combination of $\left(\vec{a}_{1}, b_{1}\right), \ldots,\left(\vec{a}_{l}, b_{l}\right)$, and thus for some $i$ we must have $\vec{a}_{i} \cdot \mathbf{x}-\vec{a}_{i} \cdot \mathbf{x}^{\prime}>0$, otherwise we would have $\vec{a} \cdot \mathbf{x}-\vec{a} \cdot \mathbf{x}^{\prime}=0$.

EXAMPLE 3.3. Consider the $S L C$ loop (1), whose transition polyhedron is $\mathcal{Q}=$ $\left\{x_{1} \geq-x_{3}, x_{1}^{\prime}=x_{1}+x_{2}, x_{2}^{\prime}=x_{2}+x_{3}, x_{3}=x_{3}^{\prime}-1\right\}$. The generators of $\operatorname{proj}_{\mathbf{x}}(\mathcal{Q})^{\#}$ are $\{(1,0,1,0),(0,0,0,1)\}$. That is, the corresponding nonnegative functions are $\rho_{1}\left(x_{1}, x_{2}, x_{3}\right)=$ $x_{1}+x_{3}$ and $\rho_{2}\left(x_{1}, x_{2}, x_{3}\right)=1$ (recall that, in each generator, the last component corresponds to the free constant $b$, and the rest to $\vec{a})$. Computing $F(\mathcal{Q})$ results in:

$$
\mathcal{Q}^{\prime}=\mathcal{Q} \wedge \Delta \rho_{1}\left(\mathbf{x}^{\prime \prime}\right) \leq 0 \wedge \Delta \rho_{2}\left(\mathbf{x}^{\prime \prime}\right) \leq 0=\mathcal{Q} \wedge\left(x_{1}+x_{3}\right)-\left(x_{1}^{\prime}+x_{3}^{\prime}\right) \leq 0
$$

This eliminates any transition for which the quantity $x_{1}+x_{3}$ decreases.

In what follows we aim at showing that $\mathcal{Q}$ has a $M \Phi R F$ of optimal depth $d$ iff $F^{d}(\mathcal{Q})=\emptyset$. We first state some auxiliary lemmas.

LEMMA 3.4. If $\mathcal{Q}^{\prime}=F(\mathcal{Q})$ has a $M \Phi R F$ of depth at most d, then $\mathcal{Q}$ has a $M \Phi R F$ of depth at most $d+1$.

Proof. Consider the generators $\left(\vec{a}_{1}, b_{1}\right), \ldots,\left(\vec{a}_{l}, b_{l}\right)$ used in Definition 3.2 , and let $\rho_{i}(\mathbf{x})=$ $\vec{a}_{i} \cdot \mathbf{x}+b_{i}$. We have $\mathcal{Q}^{\prime}=\mathcal{Q} \wedge \Delta \rho_{1}\left(\mathbf{x}^{\prime \prime}\right) \leq 0 \wedge \cdots \wedge \Delta \rho_{l}\left(\mathbf{x}^{\prime \prime}\right) \leq 0$. We assume that no $\rho_{i}$ is redundant, otherwise we take an irredundant subset. Note that simply appending $\rho_{1}, \ldots, \rho_{l}$ to a M $\Phi \mathrm{RF} \tau$ of $\mathcal{Q}^{\prime}$ does not always produce a M $\Phi \mathrm{RF}$, since the components of $\tau$ are not guaranteed to decrease over $\mathcal{Q} \backslash \mathcal{Q}^{\prime}$. Let $\tau=\left\langle g_{1}, \ldots, g_{d}\right\rangle$ be a M $\Phi$ RF for $\mathcal{Q}^{\prime}$, and w.l.o.g. assume that it is of optimal depth, we show how to construct a M $\Phi \mathrm{RF}$ $\left\langle g_{1}^{\prime}+1, \ldots, g_{d}^{\prime}+1, g_{d+1}\right\rangle$ for $\mathcal{Q}$. We construct the components one by one.

If $g_{1}$ is decreasing over $\mathcal{Q}$, we define $g_{1}^{\prime}(\mathbf{x})=g_{1}(\mathbf{x})$, otherwise we have

$$
\begin{aligned}
& \mathbf{x}^{\prime \prime} \in \mathcal{Q} \rightarrow \Delta \rho_{1}\left(\mathbf{x}^{\prime \prime}\right)>0 \vee \cdots \vee \Delta \rho_{l}\left(\mathbf{x}^{\prime \prime}\right)>0 \vee \Delta g_{1}\left(\mathbf{x}^{\prime \prime}\right)-1 \geq 0 \\
& \mathbf{x}^{\prime \prime} \in \mathcal{Q} \not \rightarrow \Delta \rho_{1}\left(\mathbf{x}^{\prime \prime}\right)>0 \vee \cdots \vee \Delta \rho_{l}\left(\mathbf{x}^{\prime \prime}\right)>0
\end{aligned}
$$


and by Lemma 2.2 there are non-negative constants $\mu_{1}, \ldots, \mu_{l}$ such that

$$
\mathbf{x}^{\prime \prime} \in \mathcal{Q} \rightarrow \Delta g_{1}\left(\mathbf{x}^{\prime \prime}\right)-1+\sum_{i=1}^{l} \mu_{i} \Delta \rho_{i}\left(\mathbf{x}^{\prime \prime}\right) \geq 0 .
$$

Define $g_{1}^{\prime}(\mathbf{x})=g_{1}(\mathbf{x})+\sum_{i=1}^{l} \mu_{i} \rho_{i}(\mathbf{x})$. Clearly we have $\mathbf{x}^{\prime \prime} \in \mathcal{Q} \rightarrow \Delta g_{1}^{\prime}\left(\mathbf{x}^{\prime \prime}\right) \geq 1$. Note also that, since $\rho_{1}, \ldots, \rho_{l}$ are non-negative on all enabled states, $g_{1}^{\prime}$ is non-negative on the states on which $g_{1}$ is non-negative. If $d>1$, we proceed with

$$
\mathcal{Q}^{(1)}=\mathcal{Q} \cap\left\{\mathbf{x}^{\prime \prime} \mid g_{1}^{\prime}(\mathbf{x}) \leq(-1)\right\} .
$$

If $g_{2}$ is decreasing over $\mathcal{Q}^{(1)}$, let $g_{2}^{\prime}=g_{2}$, otherwise, since transitions in $\mathcal{Q}^{\prime} \cap \mathcal{Q}^{(1)}$ are ranked by $\left\langle g_{2}, \ldots, g_{d}\right\rangle$ we have

$$
\begin{aligned}
& \mathbf{x}^{\prime \prime} \in \mathcal{Q}^{(1)} \rightarrow \Delta \rho_{1}\left(\mathbf{x}^{\prime \prime}\right)>0 \vee \cdots \vee \Delta \rho_{l}\left(\mathbf{x}^{\prime \prime}\right)>0 \vee \Delta g_{2}\left(\mathbf{x}^{\prime \prime}\right)-1 \geq 0 \\
& \mathbf{x}^{\prime \prime} \in \mathcal{Q}^{(1)} \not \rightarrow \Delta \rho_{1}\left(\mathbf{x}^{\prime \prime}\right)>0 \vee \cdots \vee \Delta \rho_{l}\left(\mathbf{x}^{\prime \prime}\right)>0
\end{aligned}
$$

and again by Lemma 2.2 we can construct the desired $g_{2}^{\prime}$ as we did for $g_{1}^{\prime}$. In general, for any $j \leq d$ we construct $g_{j+1}^{\prime}$ such that $\Delta g_{j+1}^{\prime}\left(\mathbf{x}^{\prime \prime}\right) \geq 1$ over

$$
\mathcal{Q}^{(j)}=\mathcal{Q} \cap\left\{\mathbf{x}^{\prime \prime} \in \mathbb{Q}^{2 n} \mid g_{1}^{\prime}(\mathbf{x}) \leq(-1) \wedge \cdots \wedge g_{j}^{\prime}(\mathbf{x}) \leq(-1)\right\}
$$

and $\mathbf{x}^{\prime \prime} \in \mathcal{Q} \wedge g_{j}(\mathbf{x}) \geq 0 \rightarrow g_{j}^{\prime}(\mathbf{x}) \geq 0$. Finally we define

$$
\mathcal{Q}^{(d)}=\mathcal{Q} \cap\left\{\mathbf{x}^{\prime \prime} \in \mathbb{Q}^{2 n} \mid g_{1}^{\prime}(\mathbf{x}) \leq(-1) \wedge \cdots \wedge g_{d}^{\prime}(\mathbf{x}) \leq(-1)\right\}
$$

and note that

$$
\mathbf{x}^{\prime \prime} \in \mathcal{Q}^{(d)} \rightarrow \Delta \rho_{1}\left(\mathbf{x}^{\prime \prime}\right)>0 \vee \cdots \vee \Delta \rho_{l}\left(\mathbf{x}^{\prime \prime}\right)>0
$$

We assume that no $\rho_{i}$ is redundant in (18), otherwise we take an irredundant subset. Now from (18) we get

$$
\mathbf{x}^{\prime \prime} \in\left(\mathcal{Q}^{(d)} \wedge \Delta \rho_{1}\left(\mathbf{x}^{\prime \prime}\right) \leq 0 \wedge \cdots \wedge \Delta \rho_{l-1}\left(\mathbf{x}^{\prime \prime}\right) \leq 0\right) \rightarrow \Delta \rho_{l}\left(\mathbf{x}^{\prime \prime}\right)>0
$$

and since the left-hand side is a polyhedron, there must be a constant $c>0$ such that

$$
\mathbf{x}^{\prime \prime} \in\left(\mathcal{Q}^{(d)} \wedge \Delta \rho_{1}\left(\mathbf{x}^{\prime \prime}\right) \leq 0 \wedge \cdots \wedge \Delta \rho_{l-1}\left(\mathbf{x}^{\prime \prime}\right) \leq 0\right) \rightarrow \Delta \rho_{l}\left(\mathbf{x}^{\prime \prime}\right) \geq c .
$$

W.l.o.g. we may assume that $c \geq 1$, otherwise we divide $\rho_{l}$ by $c$. Then we have

$$
\begin{aligned}
& \mathbf{x}^{\prime \prime} \in \mathcal{Q}^{(d)} \rightarrow \Delta \rho_{1}\left(\mathbf{x}^{\prime \prime}\right)>0 \vee \cdots \vee \Delta \rho_{l-1}\left(\mathbf{x}^{\prime \prime}\right)>0 \vee \Delta \rho_{l}\left(\mathbf{x}^{\prime \prime}\right)-1 \geq 0 \\
& \mathbf{x}^{\prime \prime} \in \mathcal{Q}^{(d)} \not \rightarrow \Delta \rho_{1}\left(\mathbf{x}^{\prime \prime}\right)>0 \vee \cdots \vee \Delta \rho_{l-1}\left(\mathbf{x}^{\prime \prime}\right)>0
\end{aligned}
$$

and again by Lemma 2.2 we can construct $g_{d+1}=\rho_{l}+\sum_{i=1}^{l-1} \mu_{i} \rho_{i}$ such that $\mathbf{x}^{\prime \prime} \in \mathcal{Q}^{(d)} \rightarrow$ $\Delta g_{d+1}\left(\mathbf{x}^{\prime \prime}\right) \geq 1$. Moreover, $g_{d+1}$ is non-negative over $\mathcal{Q}^{(d)}$ and thus it ranks all transitions in $\mathcal{Q}^{(d)}$. Now, by construction, $\tau^{\prime}=\left\langle g_{1}^{\prime}+1, \ldots, g_{d}^{\prime}+1, g_{d+1}\right\rangle$ is a $\operatorname{M} \Phi R F$ for $\mathcal{Q}$. 


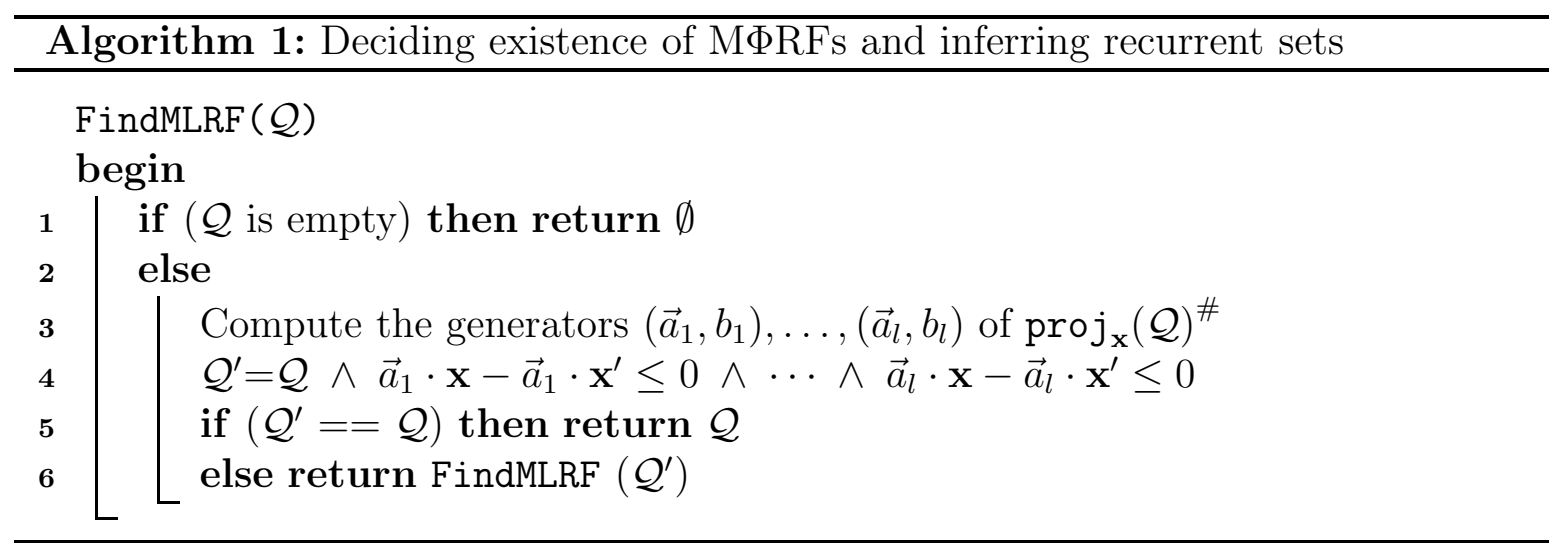

LEMMA 3.5. If $\mathcal{Q}$ has a $M \Phi R F$ of depth $d$ then $\mathcal{Q}^{\prime}=F(\mathcal{Q})$ has a $M \Phi R F$ of depth at most $d-1$.

Proof. Let $\tau=\left\langle\rho_{1}, \ldots, \rho_{k}\right\rangle$ be an $\mathrm{M} \Phi \mathrm{RF}$ for $\mathcal{Q}$, of optimal depth $k \leq d$. Without lose of generality we may assume that $\rho_{k}$ is non-negative on all $\operatorname{proj}_{\mathbf{x}}(\mathcal{Q})$, this follows immediately from the definition of nested M $M F F$ [26], which is a special case of M $\mathrm{RF}$ in which the last component is nonnegative, and the fact that for the case of $S L C$ loops existence of a M $\Phi R F$ implies the existence of a nested M $M F$ [8] of the same optimal depth. Clearly $\tau^{\prime}=\left\langle\rho_{1}, \ldots, \rho_{k-1}\right\rangle$ is a $\operatorname{M} \Phi R$ for $\mathcal{Q} \wedge \Delta \rho_{k}\left(\mathbf{x}^{\prime \prime}\right) \leq 0$ since transitions that are ranked by $\rho_{k}$ are eliminated. Now since $\rho_{k}$ is a conic combination of the generators of $\operatorname{proj}_{\mathbf{x}}(\mathcal{Q})^{\#}$ we have $\mathcal{Q}^{\prime}=F(\mathcal{Q}) \subseteq \mathcal{Q} \wedge \Delta \rho_{k}\left(\mathbf{x}^{\prime \prime}\right) \leq 0$ and thus $\tau^{\prime}$ is a M $\mathrm{RF}$ for $\mathcal{Q}^{\prime}$ as well.

LEMMA 3.6. $\mathcal{Q}$ has a $M \Phi R F$ of depth d iff $F^{d}(\mathcal{Q})=\emptyset$.

Proof. For the first direction, suppose that $\mathcal{Q}$ has a M $\Phi \mathrm{RF}$ of depth at most $d$, then applying Lemma 3.5 iteratively we must reach $F^{k}(\mathcal{Q})=\emptyset$ for some $k \leq d$, thus $F^{d}(\mathcal{Q})=$ $\emptyset$. For the other direction, suppose $F^{d}(\mathcal{Q})=\emptyset$, then using Lemma 3.4 we can construct a $\operatorname{M} \Phi \mathrm{RF}$ of depth $d$. It is easy to see that if $F^{d}(\mathcal{Q})=\emptyset$ and $F^{d-1}(\mathcal{Q}) \neq \emptyset$, then $d$ is the optimal depth.

Procedure FindMLRF $(\mathcal{Q})$ of Algorithm 1 implements the above idea, it basically applies $F$ (lines 3.4) iteratively until it either reaches an empty set (Line 1) or stabilizes (Line 5). If it returns $\emptyset$ then $\mathcal{Q}$ has a $M \Phi R F$ and we can construct one simply by invoking the polynomial-time procedure for synthesizing nested M $\Phi$ RFs as described in [8], or construct one as in the proof of Lemma 3.4. Note that, by Lemma 3.6, if we bound the recursion depth by a parameter $d$, then the algorithm is actually a decision procedure for the existence of M $\Phi$ Rs of at most depth $d$. The case in which it returns 
a nonempty set is discussed in Section 3.2. Note that the complexity of Algorithm 1 is exponential since computing the generators at Line 3 might take exponential time. In Section 5 we provide a polynomial-time implementation that does not require computing the generators.

For implementation purposes, we note that computing the generators of $\operatorname{proj}_{\mathbf{x}}(\mathcal{Q})^{\#}$ at Line 3 can be done without computing $\operatorname{proj}_{\mathbf{x}}(\mathcal{Q})$ as follows. Assume that $\mathcal{Q} \equiv$ $\left[A^{\prime \prime} \mathbf{x} \leq \mathbf{c}^{\prime \prime}\right]$, using Farkas' lemma we get that $(\vec{a}, b) \in \operatorname{proj}_{\mathbf{x}}(\mathcal{Q})^{\#}$ iff it is satisfies the constraint set $\mathcal{C} \equiv\left[\vec{\lambda} A^{\prime \prime}=(-\vec{a}, \overrightarrow{0}) \wedge \vec{\lambda} \mathbf{c} \leq b \wedge \vec{\lambda} I \geq \overrightarrow{0}\right]$, for some $\vec{\lambda}$ ( $I$ is an identity matrix of appropriate dimension). $\mathcal{C}$ defines a cone over the coordinates $\vec{\lambda}, \vec{a}$ and $b$. We can compute its generators using a standard algorithm, and then take the coordinates that correspond to $(\vec{a}, b)$.

EXAMPLE 3.7. Let us apply the algorithm to Loop (1). We start by calling FindMLRF with the transition polyhedron $\mathcal{Q}=\left\{x_{1} \geq-x_{3}, x_{1}^{\prime}=x_{1}+x_{2}, x_{2}^{\prime}=x_{2}+x_{3}, x_{3}^{\prime}=\right.$ $\left.x_{3}-1\right\}$ and proceed as follows $\left(\mathcal{Q}_{i}\right.$ represents the polyhedron passed in the $i$-th call to FindMLRF):

\begin{tabular}{|l|l|}
\hline \multicolumn{1}{|c|}{$\mathcal{Q}_{i}$} & \multicolumn{1}{c|}{ Generators of $\operatorname{proj}_{\mathbf{x}}\left(\mathcal{Q}_{i}\right)^{\#}$} \\
\hline $\mathcal{Q}_{0}=\mathcal{Q}$ & $\{(\mathbf{1}, \mathbf{0}, \mathbf{1}, \mathbf{0}),(0,0,0,1)\}$ \\
\hline $\mathcal{Q}_{1}=\mathcal{Q}_{0} \wedge\left(x_{1}+x_{3}\right)-\left(x_{1}^{\prime}+x_{3}^{\prime}\right) \leq 0$ & $\{(\mathbf{0}, \mathbf{1}, \mathbf{0},-\mathbf{1}),(1,0,1,0),(0,0,0,1)\}$ \\
\hline $\mathcal{Q}_{2}=\mathcal{Q}_{1} \wedge x_{2}-x_{2}^{\prime} \leq 0$ & $\{(\mathbf{0}, \mathbf{0}, \mathbf{1}, \mathbf{0}),(0,1,0,-1),(1,0,1,0),(0,0,0,1)\}$ \\
\hline $\mathcal{Q}_{3}=\mathcal{Q}_{2} \wedge x_{3}-x_{3}^{\prime} \leq 0=\emptyset$ & \\
\hline
\end{tabular}

Let us explain the above steps:

- $\mathcal{Q}_{0}$ is not empty, so we compute the generators of $\operatorname{proj}_{\mathbf{x}}\left(\mathcal{Q}_{0}\right)^{\#}$, which define the nonnegative functions $\rho_{1}\left(x_{1}, x_{2}, x_{3}\right)=x_{1}+x_{3}$ and $\rho_{2}\left(x_{1}, x_{2}, x_{3}\right)=1$, and then compute $\mathcal{Q}_{1}=\mathcal{Q}_{0} \wedge \Delta \rho_{1}\left(\mathbf{x}^{\prime \prime}\right) \leq 0 \wedge \Delta \rho_{2}\left(\mathbf{x}^{\prime \prime}\right) \leq 0$; and since it differs from $\mathcal{Q}_{0}$ we recursively call FindMLRF $\left(\mathcal{Q}_{1}\right)$.

- $\mathcal{Q}_{1}$ is not empty, so we compute the generators of $\operatorname{proj}_{\mathbf{x}}\left(\mathcal{Q}_{1}\right)^{\#}$, which define the nonnegative function $\rho_{3}\left(x_{1}, x_{2}, x_{3}\right)=x_{2}-1$, and then compute $\mathcal{Q}_{2}=\mathcal{Q}_{1} \wedge$ $\Delta \rho_{3}\left(\mathbf{x}^{\prime \prime}\right) \leq 0$; and since it differs from $\mathcal{Q}_{1}$ we recursively call FindMLRF $\left(\mathcal{Q}_{2}\right)$. Note that the only new generator wrt. the previous iteration is the one in bold font, the others can be ignored since they have been used already when computing $\mathcal{Q}_{1}$.

- $\mathcal{Q}_{2}$ is not empty, so we compute the generators of $\operatorname{proj}_{\mathbf{x}}\left(\mathcal{Q}_{2}\right)^{\#}$, which define the nonnegative function $\rho_{4}\left(x_{1}, x_{2}, x_{3}\right)=x_{3}$, and then compute $\mathcal{Q}_{3}=\mathcal{Q}_{2} \wedge \Delta \rho_{4}\left(\mathbf{x}^{\prime \prime}\right) \leq$ 0 ; and since it differs from $\mathcal{Q}_{2}$ we recursively call FindMLRF $\left(\mathcal{Q}_{3}\right)$.

- $\mathcal{Q}_{3}$ is empty, so we return $\emptyset$. 
Since we have reached an empty set in 3 iterations, we conclude that Loop (11) has a M $\Phi$ RF of optimal depth 3, e.g., $\left\langle x_{3}+1, x_{2}+1, x_{1}+x_{3}+1\right\rangle$.

Let us discuss now the case in which the variables range over the integers, i.e., the set of integer transitions $I(\mathcal{Q})$. It is know that $I(\mathcal{Q})$ has a $\mathrm{M} \Phi \mathrm{RF}$ iff the integer hull $\mathcal{Q}_{I}$ of $\mathcal{Q}$ has a M $\Phi$ RF (over the rationals) [8, Sect. 5]. This leads to the following lemma.

LEMMA 3.8. $I(\mathcal{Q})$ has a $M \Phi R F$ of depth d iff $F^{d}\left(\mathcal{Q}_{I}\right)=\emptyset$.

\subsection{Inference of recurrent sets}

Next we discuss the case in which $\operatorname{FindMLRF}(\mathcal{Q})$ returns a nonempty set of transition $\mathcal{S} \subseteq \mathcal{Q}$ (Line 5), and show that $\mathcal{S}$ is always a recurrent set, implying that $\mathcal{Q}$ is nonterminating. In Section [6 we discuss an experimental evaluation regards the use of Algorithm 1 for proving nontermination.

LEMMA 3.9. Let $\mathcal{S} \subseteq \mathbb{Q}^{2 n}$ be a polyhedron, if $\mathcal{S}=F(\mathcal{S})$ then $\mathcal{S}$ is a recurrent set.

Proof. According Definition 2.4 , we need to show that $\operatorname{proj}_{\mathbf{x}^{\prime}}(\mathcal{S}) \subseteq \operatorname{proj}_{\mathbf{x}}(\mathcal{S})$. Recall that since $\operatorname{proj}_{\mathbf{x}}(\mathcal{S})$ and $\operatorname{proj}_{\mathbf{x}^{\prime}}(\mathcal{S})$ are closed convex sets, each is an intersection of half-spaces that are defined by the corresponding sets $\operatorname{proj}_{\mathbf{x}}(\mathcal{S})^{\#}$ and $\operatorname{proj}_{\mathbf{x}^{\prime}}(\mathcal{S})^{\#}$, e.g., $\operatorname{proj}_{\mathbf{x}}(\mathcal{S})=\wedge\left\{\vec{a} \cdot \mathbf{x}+b \geq 0 \mid(\vec{a}, b) \in \operatorname{proj}_{\mathbf{x}}(\mathcal{S})^{\#}\right\}$. Thus, it is enough to show that $\operatorname{proj}_{\mathbf{x}}(\mathcal{S})^{\#} \subseteq \operatorname{proj}_{\mathbf{x}^{\prime}}(\mathcal{S})^{\#}$.

Let $(\vec{a}, b) \in \operatorname{proj}_{\mathbf{x}}(\mathcal{S})^{\#}$, we show that $(\vec{a}, b) \in \operatorname{proj}_{\mathbf{x}^{\prime}}(\mathcal{S})^{\#}$ as well. Define $\rho(\mathbf{x})=$ $\vec{a} \cdot \mathbf{x}+b$, which is nonnegative over $\operatorname{proj}_{\mathbf{x}}(\mathcal{S})$, and note that, by definition of $F$, since $\mathcal{S}=F(\mathcal{S})$ we have

$$
\mathbf{x}^{\prime \prime}=\left(\mathbf{x}, \mathbf{x}^{\prime}\right) \in \mathcal{S} \models 0 \leq \rho(\mathbf{x}) \leq \rho\left(\mathbf{x}^{\prime}\right)
$$

so $\vec{a} \cdot \mathbf{x}^{\prime}+b \geq 0$ holds for any $\mathbf{x}^{\prime} \in \operatorname{proj}_{\mathbf{x}^{\prime}}(\mathcal{S})$ and thus $(\vec{a}, b) \in \operatorname{proj}_{\mathbf{x}^{\prime}}(\mathcal{S})$.

Corollary 3.10. If $\operatorname{FindMLRF}(\mathcal{Q})$ returns $\mathcal{S} \neq \emptyset$ then $\mathcal{S}$ is a recurrent set, and thus $\mathcal{Q}$ is nonterminating.

Proof. This follows from the last lemma, since the algorithm returns a nonempty set $\mathcal{S} \subseteq \mathcal{Q}$ iff it finds one such that $\mathcal{S}=F(\mathcal{S})$ (Line 5 of FindMLRF)

EXAMPLE 3.11. Let us apply the algorithm to the following loop, from [35]:

$$
\text { while }\left(x_{1}-x_{2} \geq 1\right) \text { do } x_{1}^{\prime}=-x_{1}+x_{2}, x_{2}^{\prime}=x_{2}
$$


This loop does not terminate, e.g., for $x_{1}=-1, x_{2}=-2$. We call FindMLRF with $\mathcal{Q}=\left\{x_{1}-x_{2} \geq 1, x_{1}^{\prime}=-x_{1}+x_{2}, x_{2}^{\prime}=x_{2}\right\}$, and proceed as in Example 3.7.

\begin{tabular}{|l|l|}
\hline \multicolumn{1}{|c|}{$\mathcal{Q}_{i}$} & \multicolumn{1}{c|}{ Generators of $\operatorname{proj}_{\mathbf{x}}\left(\mathcal{Q}_{i}\right)^{\#}$} \\
\hline \hline $\mathcal{Q}_{0}=\mathcal{Q}$ & $\{(\mathbf{1 , - 1 , - 1 ) , ( 0 , 0 , 1 ) \}}$ \\
\hline $\mathcal{Q}_{1}=\mathcal{Q}_{0} \wedge\left(x_{1}-x_{2}\right)-\left(x_{1}^{\prime}-x_{2}^{\prime}\right) \leq 0$ & $\{(-\mathbf{2}, \mathbf{1}, \mathbf{0}),(1,-1,-1),(0,0,1)\}$ \\
\hline $\mathcal{Q}_{2}=\mathcal{Q}_{1} \wedge\left(-2 x_{1}+x_{2}\right)-\left(-2 x_{1}^{\prime}+x_{2}^{\prime}\right) \leq 0$ & $\{(\mathbf{2 , - 1 , 0}),(-\mathbf{1}, \mathbf{0},-\mathbf{1}),(-2,1,0),(0,0,1)\}$ \\
\hline $\mathcal{Q}_{3}=\mathcal{Q}_{2} \wedge\left(2 x_{1}-x_{2}\right)-\left(2 x_{1}^{\prime}-x_{2}^{\prime}\right) \leq 0 \wedge$ & \\
$\left(-x_{1}\right)-\left(-x_{1}^{\prime}\right) \leq 0$ & \\
\hline
\end{tabular}

Let us explain the above steps:

- $\mathcal{Q}_{0}$ is not empty, so we compute the generators of $\operatorname{proj}_{\mathbf{x}}\left(\mathcal{Q}_{0}\right)^{\#}$, which define the nonnegative functions $\rho_{1}\left(x_{1}, x_{2}, x_{3}\right)=x_{1}-x_{2}-1$ and $\rho_{2}\left(x_{1}, x_{2}, x_{3}\right)=1$, and then compute $\mathcal{Q}_{1}=\mathcal{Q}_{0} \wedge \Delta \rho_{1}\left(\mathbf{x}^{\prime \prime}\right) \leq 0 \wedge \Delta \rho_{2}\left(\mathbf{x}^{\prime \prime}\right) \leq 0$; and since it differs from $\mathcal{Q}_{0}$ we recursively call FindMLRF $\left(\mathcal{Q}_{1}\right)$.

- $\mathcal{Q}_{1}$ is not empty, so we compute the generators of $\operatorname{proj}_{\mathbf{x}}\left(\mathcal{Q}_{1}\right)^{\#}$, which define the nonnegative function $\rho_{3}\left(x_{1}, x_{2}, x_{3}\right)=-2 x_{1}+x_{2}$, and then compute $\mathcal{Q}_{2}=$ $\mathcal{Q}_{1} \wedge \Delta \rho_{3}\left(\mathbf{x}^{\prime \prime}\right) \leq 0$; and since it differs from $\mathcal{Q}_{1}$ we invoke FindMLRF $\left(\mathcal{Q}_{2}\right)$.

- $\mathcal{Q}_{2}$ is not empty, so we compute the generators of $\operatorname{proj}_{\mathbf{x}}\left(\mathcal{Q}_{2}\right)^{\#}$, which define the nonnegative functions $\rho_{4}\left(x_{1}, x_{2}, x_{3}\right)=2 x_{1}-x_{2}$ and $\rho_{5}\left(x_{1}, x_{2}, x_{3}\right)=-x_{1}-1$, and then compute $\mathcal{Q}_{3}=\mathcal{Q}_{2} \wedge \Delta \rho_{4}\left(\mathbf{x}^{\prime \prime}\right) \leq 0 \wedge \Delta \rho_{5}\left(\mathbf{x}^{\prime \prime}\right) \leq 0$; and since it is equal to $\mathcal{Q}_{2}$ we return $\mathcal{Q}_{2}$.

Thus, $\mathcal{Q}_{2}$ is a recurrent set of transitions and we conclude that Loop (23) is not terminating. Projecting $\mathcal{Q}_{2}$ on $x_{1}$ and $x_{2}$ we get $\left\{x_{1} \leq-1,2 x_{1}-x_{2}=0\right\}$, which is the corresponding recurrent set of states.

We remark that Loop (23) has a fixed point $(-1,-2)$, i.e., from state $x_{1}=-1, x_{2}=$ -2 we have a transition to $x_{1}=-1, x_{2}=-2$. The algorithm also detects nontermination of some loops that do not have fixed points. For example, if we change $x_{2}^{\prime}=x_{2}$ in Loop (23) by $x_{2}^{\prime}=x_{2}-1$, we get obtain a recurrent set of transitions $\mathcal{S}$ such that $\operatorname{proj}_{\mathbf{x}}(\mathcal{S})=\left\{-2 x_{2} \geq 3,4 x_{1}-2 x_{2}=1\right\}$.

Now that we have seen the possible outcomes of the algorithm (in case it terminates), we see that this approach reveals a relation between seeking M $\Phi$ RF and seeking recurrent sets. A possible view is that the algorithm seeks a recurrent set (of a particular form) and when it concludes that no such set exists, i.e., reaching $\emptyset$, we can construct a M $\Phi$ RF. 
The recurrent sets inferred by Algorithm 1 belong to a narrower class than that of Definition 2.4. In fact, for a polyhedral set $\mathcal{S}$ to be a recurrent set, Definition 2.4

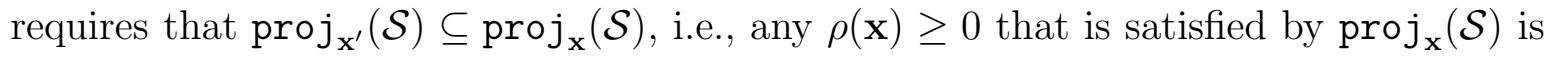
also satisfied by $\operatorname{proj}_{\mathbf{x}^{\prime}}(\mathcal{S})$. On the other hand, in our recurrent sets, $\rho$ is monotonic as well, i.e., $\rho\left(\mathbf{x}^{\prime}\right) \geq \rho(\mathbf{x})$ for any $\left(\mathbf{x}, \mathbf{x}^{\prime}\right) \in \mathcal{S}$.

EXAMPLE 3.12. Consider the following $S L C$ loop:

$$
\text { while }(x \geq 0) \text { do } x^{\prime}=1-x
$$

The largest recurrent set of transitions for this loop is $\left\{x \geq 0, x \leq 1, x^{\prime}=1-x\right\}$, and Algorithm 1 infers $\left\{x=\frac{1}{2}, x^{\prime}=\frac{1}{2}\right\}$. In the first iteration it eliminates all transitions for which $x-x^{\prime}>0$, i.e., those for which $x \in\left(\frac{1}{2}, \infty\right)$, and in the second those for which $(-x)-\left(-x^{\prime}\right)>0$, i.e., those for which $x \in\left[0, \frac{1}{2}\right)$. Note that this is the largest monotonic recurrent set.

At this point, it is natural to explore in the difference between the different notions of recurrent sets. The most intriguing question is if nonterminating $S L C$ loops always have monotonic recurrent sets (either polyhedral or closed convex in general). This is clearly true for loops that have a fixed point, i.e., there is $\mathbf{x}$ such that $(\mathbf{x}, \mathbf{x}) \in \mathcal{Q}$, however, this question is left open for the general case. We note that the geometric nontermination argument introduced in [27] is also related to monotonic recurrent sets. Specifically, it is easy to show that in some cases (when the coefficients $\mu_{i}$ and $\lambda_{i}$, in Definition 5 of [27], are either 0 or at least 1), we can construct a monotonic recurrent set from the geometric nontermination argument.

Let us discuss now the case of integer loops. First we note that the difference between the different notions of recurrent sets is clear in this case. Example 3.12 shows that the loop has a recurrent set $\{(0,1),(1,0)\}$, but does not have a monotonic recurrent set. Apart from this difference, a natural question to ask is if the recurrent set $\mathcal{S}$ returned by $\operatorname{FindMLRF}(\mathcal{Q})$, or more precisely $I(\mathcal{S})$ if it is not empty, witnesses nontermination of $I(\mathcal{Q})$. There are some cases for which this is true.

LEMMA 3.13. Let $\mathcal{Q}$ be a $S L C$ loop with affine update $\mathbf{x}^{\prime}=U \mathbf{x}+\mathbf{c}$ as in (5), and assume the coefficients $U$ and $\mathbf{c}$ are integer. If $\mathcal{S}$ is a recurrent set of $\mathcal{Q}$, and $I(\mathcal{S})$ is not empty, then $I(\mathcal{S})$ is recurrent for $I(\mathcal{Q})$.

Proof. Since the update is affine with integer coefficients, it follows that any state in $\operatorname{proj}_{\mathbf{x}}(I(\mathcal{S}))$ has a successor in $\operatorname{proj}_{\mathbf{x}^{\prime}}(I(\mathcal{S})) \subseteq \operatorname{proj}_{\mathbf{x}}(I(\mathcal{S}))$, which is the definition of a recurrent set. 
We note that one can allow some degree of non-determinism in the above definition, in particular, nondeterministically setting a variable to an arbitrary value. Note that tools for proving nontermination that are based on the use of Farkas' lemma [22, 24], impose similar restrictions to guarantee that the recurrent set is valid over the integers. The next example demonstrates that the above lemma does not apply for $S L C$ loops in general, even when the algorithm is applied to the integer hull $\mathcal{Q}_{I}$. This is because it is not guaranteed that any integer state $\mathbf{x} \in I\left(\operatorname{proj}_{\mathbf{x}}(\mathcal{S})\right)$ has an integer successor $\mathbf{x}^{\prime} \in I\left(\operatorname{proj}_{\mathbf{x}^{\prime}}(\mathcal{S})\right)$.

EXAMPLE 3.14. Consider the following loop

$$
\text { while }(x \geq 2) \text { do } x^{\prime}=\frac{3}{2} x
$$

which is clearly nonterminating over the rationals, for any $x \geq 2$. However, it is terminating over the integers because $(i)$ starting from $x$ odd, the next state $\frac{3}{2} x$ is not integer, and; ( $i i)$ starting from $x$ even, then for some $i>0$ we have $\frac{3^{i}}{2^{i}} x$ odd (because $\frac{x}{2^{i}}$ must be odd some $i>0$ and $3^{i}$ is odd), and then the next state in not integer. The algorithm returns $\mathcal{Q}$ as a recurrent set, but $I(\mathcal{Q})$, which is not empty, is not a recurrent set as the loop is terminating over the integers. Note that the transition polyhedron is integral, i.e., $\mathcal{Q}=\mathcal{Q}_{I}$.

\subsection{Cases in which Algorithm 1 does not terminate}

When Algorithm 1 terminates, it either finds a M $\Phi$ R or proves nontermination of the given loop. This means that if applied to a terminating loop that does not have a M $\Phi$ F, then Algorithm 1 does not terminate. This nonterminating behaviour shows that our algorithm is not complete, however, it can also be used to prove that a given loop does not have a M $\Phi R F$.

EXAMPLE 3.15. Consider the following loop

$$
\text { while }\left(x_{1} \geq x_{2}, x_{2} \geq 1\right) \text { do } x_{1}^{\prime}=2 x_{1}, x_{2}^{\prime}=3 x_{2}
$$

which is terminating [26]. It is easy to show (by induction) that the polyhedron passed to FindMLRF in the $i$-th call is $\mathcal{Q}_{i}=\left\{x_{1} \geq 2^{i} x_{2}, x_{2} \geq 1, x_{1}^{\prime}=2 x_{1}, x_{2}^{\prime}=3 x_{2}\right\}$, which satisfies $\mathcal{Q}_{i} \neq \emptyset$ and $\mathcal{Q}_{i+1} \subset \mathcal{Q}_{i}$. This implies that the algorithm does not terminate, and thus the loop does not have a $M \Phi R F$. Note that $\operatorname{proj}_{\mathbf{x}}\left(\mathcal{Q}_{i}\right)^{\#}$ is generated by the rays $(0,1)$ and $\left(1,-2^{i}\right)$.

The following example shows that Algorithm 1 might not terminate also when applied to nonterminating loops. 
EXAMPLE 3.16. Consider the following loop

$$
\text { while }\left(x_{1}+x_{2} \geq 3\right) \text { do } x_{1}^{\prime}=3 x_{1}-2, x_{2}^{\prime}=2 x_{2}
$$

which is nonterminating (even over integers) [27]. Algorithm 1] does not terminate on this loop. The loop does have a monotonic recurrent set, e.g., $\mathcal{S}=\left\{x_{1} \geq 1\right.$, $\left.x_{2}^{\prime}=2 x_{2}, x_{1}^{\prime}=3 x_{1}-2\right\}$.

When the algorithm does not terminate, the iterates $F^{i}(\mathcal{Q})$ converge to $\mathcal{Q}_{\omega}=$ $\cap_{i \geq 0} F^{i}(\mathcal{Q})$. For example, for Loop (26), which is terminating, we have $\mathcal{Q}_{\omega}=\emptyset$, and for Loop (27), which is not terminating, we have $\mathcal{Q}_{\omega}=\left\{x_{1} \geq 1, x_{2}^{\prime}=2 x_{2}, x_{1}^{\prime}=3 x_{1}-2\right\}$ which is a monotonic recurrent set. Some natural questions arise at this point: $(i)$ is it true that $\mathcal{Q}_{\omega}=\emptyset$ iff $\mathcal{Q}$ is terminating? (ii) is it a true that if $\mathcal{Q}_{\omega} \neq \emptyset$ then it is a (monotonic) recurrent set? We note that for deterministic loops, termination implies $\mathcal{Q}_{\omega}=\emptyset$, and it also not hard to show that if $\mathcal{Q}_{\omega} \neq \emptyset$ then $\mathcal{Q}_{\omega}$ is a monotonic recurrent set. The general questions are left open.

Once we start to explore properties of $\mathcal{Q}_{\omega}$, there is a difference between real and rational loops as we demonstrate in the next example.

EXAMPLE 3.17. Consider the following loop

$$
\text { while }(4 x+y \geq 1) \text { do } x^{\prime}=-2 x+4 y, y^{\prime}=4 x
$$

which is terminating over the rationals and nonterminating over the reals [12]. The algorithm does not terminate when applied to this loop. If each $\mathcal{Q}_{i}$ is considered as a set of rational transitions, then $\mathcal{Q}_{w}=\emptyset$, however, if we include also the irrational transitions then $\mathcal{Q}_{w}$ would be the closed convex set $\left\{c \cdot\left(\sqrt{17}-1,4,(\sqrt{17}-1)^{2}, 4(\sqrt{17}-1)\right) \mid c \geq\right.$ $\left.\frac{1}{\sqrt{17}+3}\right\}$, which is a monotonic recurrent set.

\section{Loops for which M $\Phi$ RFs are sufficient}

The purpose of this section is to demonstrate the usefulness of Algorithm 1 for studying properties of $S L C$ loops. In particular, we use it characterize kinds of $S L C$ loops for which there is always a $M \Phi R F$, if the loop is terminating. We shall prove this result for two kinds of loops, both considered in previous work, namely octagonal relations and affine relations with the finite-monoid property - for both classes, termination has been proven decidable in [10]. We only consider the rational case. Another question, which we do not answer, is whether we can ensure that Algorithm 1 recognizes the non-terminating members of the class. 
Let us set some notation first. The composition of transition relations $S, T \subseteq \mathbb{Q}^{2 n}$ is defined as $S \circ T=\left\{\left(\begin{array}{l}\mathbf{x} \\ \mathbf{z}\end{array}\right) \mid \exists \mathbf{y} \cdot\left(\begin{array}{l}\mathbf{x} \\ \mathbf{y}\end{array}\right) \in S \wedge\left(\begin{array}{l}\mathbf{y} \\ \mathbf{z}\end{array}\right) \in T\right\}$. We let $T^{n}=T^{n-1} \circ T$ where $T^{0}$ is the identity relation. We use $\operatorname{pre}^{n}(T)$ for the projection of $T^{n}$ over the first $n$ coordinates, i.e., $\operatorname{pre}^{n}(T)=\operatorname{proj}_{\mathbf{x}}\left(T^{n}\right)$, which is the set of states from which we can make traces of length at least $n$ (for nondeterministic loops some might be less than $n$ as well). When $T$ is polyhedral, i.e., a $S L C$ loop, then $T^{n}$ and $\operatorname{pre}^{n}(T)$ are polyhedral as well.

\subsection{Finite loops}

First, we consider loops which always terminate and, moreover, their number of iterations is bounded by a constant, i.e., there is $N>0$ such that $\mathcal{Q}^{N}=\emptyset$. Note that such loop terminates in at most $N-1$ iterations, or equivalently $N-1$ is an upper-bound on the length of the corresponding traces.

LEMMA 4.1. If $\mathcal{Q}^{N}=\emptyset$, then it has a $M \Phi R F$ of depth less than $N$.

Proof. The proof is by induction on $N$. For $N=1, \mathcal{Q}=\emptyset$, and it has a M $\Phi$ RF of zero depth, by definition. Let $N>1$, and assume that $\mathcal{Q}^{N-1} \neq \emptyset$, otherwise it trivially follows for $N$. Consider a transition $\mathbf{x}^{\prime \prime}=\left(\mathbf{x}, \mathbf{x}^{\prime}\right)$ that is the last in a terminating trace. We have $\mathbf{x} \in \operatorname{proj}_{\mathbf{x}}(\mathcal{Q})$ and $\mathbf{x}^{\prime} \notin \operatorname{proj}_{\mathbf{x}}(\mathcal{Q})$. Since $\operatorname{proj}_{\mathbf{x}}(\mathcal{Q})$ is a closed polyhedral set, this means that there is a function $\rho$, defined by some $(\vec{a}, b) \in \operatorname{proj}_{\mathbf{x}}(\mathcal{Q})^{\#}$, that is nonnegative over $\operatorname{proj}_{\mathbf{x}}(\mathcal{Q})$ but negative on $\mathbf{x}^{\prime}$, and thus $\Delta \rho\left(\mathbf{x}^{\prime \prime}\right)=\rho(\mathbf{x})-\rho\left(\mathbf{x}^{\prime}\right)>0$. It follows that $\mathbf{x}^{\prime \prime}$ is eliminated by Algorithm 1 when computing $\mathcal{Q}^{\prime}$ at Line 4. This means that any transition of $\mathcal{Q}^{\prime}$ cannot be the last transition of any terminating run of $\mathcal{Q}$, and thus $\left(\mathcal{Q}^{\prime}\right)^{N-1}=\emptyset$. Therefore, by induction, it has a M $\Phi$ RF of depth at most $N-2$, and by Lemma $3.5 \mathcal{Q}$ has a $M \Phi R F$ of depth at most $N-1$.

\subsection{The class $\mathrm{RF}(b)$}

This class contains loops which can be described as having the following behavior: Transitions are linearly ranked, as long as we are in states from which we can make runs of length at least $b$. In other words, once we reach a state from which we cannot make more than $b-1$ transitions we do not require the rest of the trace to be linearly ranked.

Definition 4.2. We say that a $S L C$ loop $\mathcal{Q}$ belongs to the class $\mathrm{RF}(b)$ if the loop $\mathcal{Q} \cap\left\{\left(\mathbf{x}, \mathbf{x}^{\prime}\right) \in \mathbb{Q}^{2 n} \mid \mathbf{x} \in \operatorname{pre}^{b}(\mathcal{Q})\right\}$ has a $L R F$.

We note that that $R F(1)$ is the class of loops which have a $L R F$. 
LEMMA 4.3. Loops in $\mathrm{RF}(b)$ have $M \Phi R F$ s of depth at most $b$.

Proof. This lemma actually generalizes Lemma 4.1, since the loops concerned there are $\operatorname{RF}(N-1)$. The proof is done similarly by induction on $b$. For $b=1, \mathcal{Q}$ has a $L R F$ by definition. Let $b>1$, and suppose that $\mathbf{x}^{\prime \prime}=\left(\mathbf{x}, \mathbf{x}^{\prime}\right) \in \mathcal{Q}$ is a last transition of a terminating run, then $\mathbf{x} \in \operatorname{proj}_{\mathbf{x}}(\mathcal{Q})$ and $\mathbf{x}^{\prime} \notin \operatorname{proj}_{\mathbf{x}}(\mathcal{Q})$. Since $\operatorname{proj}_{\mathbf{x}}(\mathcal{Q})$ is a closed polyhedral set, this means that there is a function $\rho$, defined by some $(\vec{a}, b) \in$ $\operatorname{proj}_{\mathbf{x}}(\mathcal{Q})^{\#}$, that is nonnegative over $\operatorname{proj}_{\mathbf{x}}(\mathcal{Q})$ but negative over $\mathbf{x}^{\prime}$, and thus $\Delta \rho\left(\mathbf{x}^{\prime \prime}\right)=$ $\rho(\mathbf{x})-\rho\left(\mathbf{x}^{\prime}\right)>0$. It follows that $\mathbf{x}^{\prime \prime}$ is eliminated by Algorithm 1 when computing $\mathcal{Q}^{\prime}$ at Line 4. This means that any transition of $\mathcal{Q}^{\prime}$ cannot be the last transition of any terminating run of $\mathcal{Q}$, and thus $\mathcal{Q}^{\prime}$ is $\operatorname{RF}(b-1)$. Therefore, by induction, it has a $M \Phi R F$ of depth at most $b-1$, and by Lemma $3.5 \mathcal{Q}$ has a M $\mathrm{MRF}$ of depth at most $b$.

EXAMPLE 4.4. Consider the loop (taken from [10]) defined by $\mathcal{Q}=\left\{x_{2}-x_{1}^{\prime} \leq-1\right.$, $\left.x_{3}-x_{2}^{\prime} \leq 0, x_{1}-x_{3}^{\prime} \leq 0, x_{4}^{\prime}-x_{4} \leq 0, x_{3}^{\prime}-x_{4} \leq 0\right\}$. This loop is $\mathrm{RF}(3)$, since adding $\operatorname{pre}^{3}(Q)=\left\{x_{2}+x_{4} \geq 1, x_{3}+x_{4} \geq 1, x_{1}+x_{4} \geq 0\right\}$ to the loop we find a $L R F$, e.g., $\rho(\mathbf{x})=-x_{1}-x_{2}-x_{3}+3 x_{4}+1$. Indeed, $\mathcal{Q}$ has a $M \Phi R F$ of optimal depth 3, e.g., $\left\langle-x_{1}-x_{2}-x_{3}+3 x_{4}+1,-\frac{2}{3} x_{1}-\frac{1}{3} x_{2}+x_{4}+1,-\frac{1}{4} x_{1}+\frac{1}{4} x_{4}+1\right\rangle$. Note that the first component is the $L R F$ that we have found above for $\mathcal{Q} \cap\left\{\mathrm{x}^{\prime \prime} \mid \mathrm{x} \in \operatorname{pre}^{3}(Q)\right\}$.

Note that if we know that a given class of loop belongs to $\mathrm{RF}(b)$, then bounding the recursion depth of Algorithm 1 by $b$ gives us a decision procedure for the existence of M $\Phi R F$ for this class. Bozga, Iosif and Konecný [10] prove that octagonal relations are $\operatorname{RF}\left(5^{2 n}\right)$, where $n$ is the number of variables 1 . Thus for octagonal relations, we can decide termination and for terminating loops obtain M $\Phi R F$. For the depth of the $\mathrm{M} \Phi \mathrm{RF}$, namely the parameter $b$ above, [10] gives a tighter (polynomial) result for those octagonal relations which allow arbitrarily long executions (called $*$-consistent).

\subsection{Loops with affine-linear updates}

In certain cases, we can handle loops with affine-linear updates - which are, in general, not octagonal. Recall that a loop with affine-linear update has a transition relation of the form:

$$
\mathcal{Q} \equiv\left[B \mathbf{x} \leq \mathbf{b} \wedge \mathbf{x}^{\prime}=U \mathbf{x}+\mathbf{c}\right] .
$$

We keep the meaning of the symbols $U, B, \mathbf{b}, \mathbf{c}$ fixed for the sequel. Moreover, we express the loop using the transformation $\mathcal{U}(\mathbf{x})=U \mathbf{x}+\mathbf{c}$ and the guard $\mathcal{G} \equiv[B \mathbf{x} \leq \mathbf{b}]$.

\footnotetext{
${ }^{1}$ Technically, they prove it just for integer loops, but the result applies to the rational case as well (one only has to simplify some considerations away from the proof).
} 
We use $U_{i j}$ to denote the entry of matrix $U$ in row $i$ and column $j$, and for a vector $\mathbf{v}$ we let $\mathbf{v}[i . . j]$ be the vector obtained from components $i$ to $j$ of the vector $\mathbf{v}$.

Our goal is to show that if $U^{p}$, for some $p>0$, is diagonalizable and all its eigenvalues are in $\{0,1\}$, then $\mathcal{Q}$ is $\operatorname{RF}(3 p)$, and thus, by Lemma 4.3, if terminating, it has a M $\Phi \mathrm{RF}$. Affine loops with the finite monoid property that has been addressed in [10], satisfy this condition (interestingly, in Section 5 we show a similar result when $U-I$ has the property). We state some auxiliary lemmas first.

LEMMA 4.5. Let $\mathcal{Q}$ be an affine-linear loop as in (29) such that, for some $N>0$, $\mathcal{Q}^{N}$ is $\operatorname{RF}(b)$. Then $\mathcal{Q}$ is $\operatorname{RF}(N(b+1))$.

Proof. If $\mathcal{Q}^{N}$ is $\operatorname{RF}(b)$, then $\mathcal{Q}^{N} \cap\left\{\mathbf{x}^{\prime \prime} \mid \mathbf{x} \in \operatorname{pre}^{b}\left(\mathcal{Q}^{N}\right)\right\}$ has a $L R F \rho$, and thus

$$
\mathbf{x} \in \operatorname{pre}^{b}\left(\mathcal{Q}^{N}\right)=\operatorname{pre}^{N b}(\mathcal{Q}) \Rightarrow \rho(\mathbf{x}) \geq 0 \wedge \rho(\mathbf{x})-\rho\left(\mathcal{U}^{N}(\mathbf{x})\right)>0 .
$$

Note that $\rho(\mathbf{x})-\rho\left(\mathcal{U}^{N}(\mathbf{x})\right)$ can be written as

$$
\sum_{j=0}^{N-1} \rho\left(\mathcal{U}^{j}(\mathbf{x})\right)-\sum_{j=0}^{N-1} \rho\left(\mathcal{U}^{j+1}(\mathbf{x})\right)
$$

This is because every term $\rho\left(\mathcal{U}^{i}(\mathbf{x})\right)$, except for $i=0$ and $i=N$, appear in (31) with positive and negative signs. Hence, if we let $\rho_{1}(\mathbf{x})=\sum_{j=0}^{N-1} \rho\left(\mathcal{U}^{j}(\mathbf{x})\right)$ then:

$$
\mathbf{x} \in \operatorname{pre}^{N b}(\mathcal{Q}) \Rightarrow \rho_{1}(\mathbf{x})-\rho_{1}(\mathcal{U}(\mathbf{x}))>0 .
$$

Moreover, $\rho_{1}$ is the sum of terms $\rho\left(\mathcal{U}^{i}(\mathbf{x})\right)$ which are bounded from below on pre ${ }^{N(b+1)}(\mathcal{Q})$. Hence, we have a $L R F$ for $\mathcal{Q} \cap\left\{\mathbf{x}^{\prime \prime} \mid \mathbf{x} \in \operatorname{pre}^{N(b+1)}(\mathcal{Q})\right\}$ and thus $\mathcal{Q}$ is $\operatorname{RF}(N(b+1))$.

LEMMA 4.6. Let $\mathcal{Q}$ be a loop as in (29), and assume $U$ is diagonal with entries in $\{0,1\}$. Then, if $\mathcal{Q}$ is terminating, it is $\mathrm{RF}(2)$.

Proof. Without loss of generality we may assume that $U_{11}=\cdots=U_{k k}=1$ and $U_{j j}=0$ for $j>k$, otherwise we could reorder the variables to put it into this form. Clearly, the update adds $\mathbf{c}_{1}=\mathbf{c}[1 . . k]$ to the first $k$ elements of $\mathbf{x}$, and sets the rest to $\mathbf{c}_{2}=\mathbf{c}[k+1 . . n]$. Consequently, such a loop is non-terminating iff the space $V=$ $\left\{\mathbf{x} \in \mathbb{Q}^{n} \mid \mathbf{x}[k+1 . . n]=\mathbf{c}_{2}\right\}$ intersects the loop guard $\mathcal{G} \equiv[B \mathbf{x} \leq \mathbf{b}]$, and the vector $\mathbf{u}=\left(c_{1}, \ldots c_{k}, 0, \ldots, 0\right)^{\mathrm{T}}$ is a recession direction of the guard, i.e., $B \mathbf{u} \leq \mathbf{0}$. To see this: suppose these conditions hold, then starting from any state $\mathbf{x}_{0} \in V$, the state after $i$ iterations will be $\mathbf{x}_{i}=\mathbf{x}_{0}+i \mathbf{u}$, which is in $\mathcal{G}$ since $\mathbf{x}_{0} \in \mathcal{G}$ and $\mathbf{u} \in$ rec.cone $(\mathcal{G})$, and thus the execution does not terminate; for the other direction, suppose it does not 
terminate, then there must be a nonterminating execution that starts in $\mathbf{x}_{0} \in V$, this execution generates the states $\mathbf{x}_{0}+i \mathbf{u} \in \mathcal{G}$ and thus $\mathbf{u}$ is a recession directions of $\mathcal{G}$.

Now suppose the loop is terminating, we show that it is $\operatorname{RF}(2)$. Let us analyze a run of the loop starting with some valid transition $\left(\mathbf{x}_{0}, \mathbf{x}_{1}\right)$. We have two cases:

1. If $\mathbf{x}_{1} \notin \mathcal{G}$, then the run terminates in 1 iteration.

2. If $\mathbf{x}_{1} \in \mathcal{G}$, then $V$ intersects with $\mathcal{G}$, since $\mathbf{x}_{1}[k+1 . . n]=\mathbf{c}_{2}$, and thus $B \mathbf{u} \leq \mathbf{0}$ should not hold, otherwise the loop is nonterminating. This means that there is a constraint $\vec{b}_{i} \cdot \mathbf{x} \leq b_{i}$ of the guard such that $\vec{b}_{i} \cdot \mathbf{u}>0$. Let $\vec{a}$ be as $\vec{b}_{i}$ but setting components $k+1 . . n$ to zero, we still have $\vec{a} \cdot \mathbf{u}>0$ because these components are 0 in $\mathbf{u}$. We show that this trace is linearly ranked by $\rho(\mathbf{x})=\vec{a} \cdot \mathbf{x}+\max \left(b_{i}, 0\right)$. Suppose the initial state is $\mathbf{x}_{0}$, and write it as $\left(\begin{array}{l}\mathbf{x}_{0}[1 . . k] \\ \mathbf{x}_{0}[k+1 . . n]\end{array}\right)$. Consider a trace that starts in $\mathbf{x}_{0}$, it is easy to see that the $i$-th state, for $i \geq 1$, is $\mathbf{x}_{i}=\left(\begin{array}{l}\mathbf{x}_{0}[1 . . k]+i \mathbf{c}_{\mathbf{1}} \\ \mathbf{c}_{2}\end{array}\right)$. Then, we have $\rho\left(\mathbf{x}_{i}\right)-\rho\left(\mathbf{x}_{i+1}\right)=\vec{a} \cdot \mathbf{u}>0$, moreover $\rho$ is nonnegative on all state except the last of the trace (which is not in the guard).

This analysis implies that any terminating trace is either of length 1 , or has a $L R F$ $\rho(\mathbf{x})=\vec{a} \cdot \mathbf{x}+\max \left(b_{i}, 0\right)$, and together with the fact the the loop is deterministic we conclude that it is $\mathrm{RF}(2)$.

Now we are in a position for proving our main result of this section.

LEMMA 4.7. If $U^{p}$, for some $p>0$, is diagonalizable and all its eigenvalues are in $\{0,1\}$, then loop (29) is either nonterminating or $\mathrm{RF}(3 p)$.

Proof. Recall that the update is $\mathcal{U}(\mathbf{x})=U \mathbf{x}+\mathbf{c}$, then $\mathcal{U}^{p}(\mathbf{x})=U^{p} \mathbf{x}+\mathbf{v}$, for a vector $\mathbf{v}=\left(I+U+\cdots+U^{p-1}\right) \mathbf{c}$. Taking into account the guard,

$$
\mathcal{Q}^{p} \equiv\left(B \mathbf{x} \leq \mathbf{b} \wedge \cdots \wedge B \mathcal{U}^{p-1}(\mathbf{x}) \leq \mathbf{b}\right) \wedge \mathbf{x}^{\prime}=\mathcal{U}^{p}(\mathbf{x})
$$

We write this guard concisely with the notation $B^{\langle p\rangle} \mathbf{x} \leq \mathbf{b}^{\langle p\rangle}$. Since, by assumption, $U^{p}$ is diagonalizable, there is a non-singular matrix $P$ and a diagonal matrix $D$ such that $P^{-1} U^{p} P=D$ and $D$ has only 1's and 0 's on the diagonal ( $P$ is a change-of-basis transformation). We consider a loop $\widehat{\mathcal{Q}}^{p}$ which is similar to $\mathcal{Q}^{p}$ but transformed by $P$, that is:

$$
\widehat{\mathcal{Q}^{p}} \equiv B P^{\langle p\rangle} \mathbf{x} \leq \mathbf{b}^{\langle p\rangle} \wedge \mathbf{x}^{\prime}=D \mathbf{x}+P^{-1} \mathbf{v}
$$

Properties like termination and linear ranking are not affected by such a change of basis. 
This is because if $\left(\mathbf{x}_{0}, \mathbf{x}_{1}\right)$ is a transition of $\mathcal{Q}^{p}$ then $\left(P^{-1} \mathbf{x}_{0}, P^{-1} \mathbf{x}_{1}\right)$ is a transition of $\widehat{\mathcal{Q}^{p}}$, and if $\left(\mathbf{x}_{0}, \mathbf{x}_{1}\right)$ is a transition of $\widehat{\mathcal{Q}^{p}}$ then $\left(P \mathbf{x}_{0}, P \mathbf{x}_{1}\right)$ is a transition of $\mathcal{Q}^{p}$. This means that there is a one-to-one correspondence between the traces. Moreover, if function $\vec{a} \cdot \mathbf{x}+b$ ranks a transition of $\mathcal{Q}^{p}$ then $\left(\vec{a} P^{-1}\right) \cdot \mathbf{x}+b$ ranks the corresponding transition of $\widehat{\mathcal{Q}^{p}}$, and if it ranks a transition $\widehat{\mathcal{Q}^{p}}$ then $(\vec{a} P) \cdot \mathbf{x}+b$ ranks the corresponding transition of $\mathcal{Q}^{p}$. We conclude that, if terminating, $\mathcal{Q}^{p}$ is $\operatorname{RF}(b)$ iff $\widehat{\mathcal{Q}^{p}}$ is $\operatorname{RF}(b)$.

Now, $\widehat{\mathcal{Q}^{p}}$ has the diagonal form discussed in Lemma 4.6, and thus, in the case that it terminates, it is $\operatorname{RF}(2)$ and so is $\mathcal{Q}^{p}$. Then using Lemma 4.5 we conclude that $\mathcal{Q}$ is $\mathrm{RF}(3 p)$.

\section{$5 \quad \mathrm{M} \Phi \mathbf{R F s}$ and the displacement polyhedron}

In this section we introduce an alternative representation for $S L C$ loops, that we refer to as the displacement polyhedron, and show that Algorithm 1, or more precisely the check $F^{k}(\mathcal{Q})=\emptyset$, has a simple encoding in this representation that can be preformed in polynomial time. Note that we already know that deciding the existence of a M $\Phi$ RF of depth $d$ can be done in polynomial time [8], so in this sense we do not provide any new knowledge. However, the construction is interesting by itself, and, apart from allowing the efficient encoding of the check $F^{k}(\mathcal{Q})=\emptyset$, it provides us with a new approach to the problem of existence of M $\Phi$ RFs in general (without a depth bound), that is still open, and other problems related to termination and nontermination of $S L C$ loops. As an indication of their utility, we show that some nontrivial observations about $S L C$ loops that are made straightforward through this representation (see the end of this section).

Definition 5.1. Given a $S L C$ loop $\mathcal{Q} \subseteq \mathbb{Q}^{2 n}$, we define its corresponding displacement polyhedron as $\mathcal{R}=\operatorname{proj}_{\mathbf{x}, \mathbf{y}}\left(\mathcal{Q} \wedge \mathbf{x}^{\prime}=\mathbf{x}+\mathbf{y}\right) \subseteq \mathbb{Q}^{2 n}$.

Note that the projection drops $\mathbf{x}^{\prime}$. Intuitively, an execution step using $\mathcal{Q}$ starts from a state $\mathbf{x}$, and chooses a state $\mathbf{x}^{\prime}$ such that $\left(\begin{array}{l}\mathbf{x} \\ \mathbf{x}^{\prime}\end{array}\right) \in \mathcal{Q}$. To perform the step using $\mathcal{R}$, select $\mathbf{y}$ such that $\left(\begin{array}{l}\mathbf{x} \\ \mathbf{y}\end{array}\right) \in \mathcal{R}$ and let the new state be $\mathbf{x}+\mathbf{y}$. By definition, we obtain the same transitions. The constraint representation of $\mathcal{R}$ can be derived from that of $\mathcal{Q}$ as follows. Let $\mathcal{Q} \equiv\left[A^{\prime \prime}\left(\begin{array}{l}\mathbf{x} \\ \mathbf{x}^{\prime}\end{array}\right) \leq \mathbf{c}^{\prime \prime}\right]$ where $A^{\prime \prime}$ is the matrix below on the left (see Section 2.2$)$, then $\mathcal{R} \equiv\left[R\left(\begin{array}{l}\mathbf{x} \\ \mathbf{y}\end{array}\right) \leq \mathbf{c}^{\prime \prime}\right]$ where $R$ is the matrix below on the right:

$$
A^{\prime \prime}=\left(\begin{array}{cc}
B & 0 \\
A & A^{\prime}
\end{array}\right) \quad R=\left(\begin{array}{cc}
B & 0 \\
A+A^{\prime} & A^{\prime}
\end{array}\right)
$$

Next, we show that the displacement polyhedron $\mathcal{R}_{k}$ of $\mathcal{Q}_{k}=F^{k}(\mathcal{Q})$ is equivalent 
to the following polyhedron projected on $\mathbf{x}$ and $\mathbf{y}_{0}$

$$
R\left(\begin{array}{l}
\mathbf{x} \\
\mathbf{y}_{0}
\end{array}\right) \leq \mathbf{c}^{\prime \prime} \wedge R\left(\begin{array}{l}
\mathbf{y}_{0} \\
\mathbf{y}_{1}
\end{array}\right) \leq \mathbf{0} \wedge \ldots \wedge R\left(\begin{array}{l}
\mathbf{y}_{k-1} \\
\mathbf{y}_{k}
\end{array}\right) \leq \mathbf{0}
$$

Now since, by Definition 5.1, $\mathcal{Q}_{k}$ is empty iff $\mathcal{R}_{k}$ is empty, the check $F^{k}(\mathcal{Q})=\emptyset$ is reduced to checking that (35) is empty, which can be done in polynomial time in the bit-size of the constraint representation of $\mathcal{Q}$ and the parameter $k$. We first show how $\mathcal{R}_{k+1}$ can be obtained from $\mathcal{R}_{k}$ similarly to $\mathcal{Q}_{k+1}=F\left(\mathcal{Q}_{k}\right)$.

LEMMA 5.2. Let $\left(\vec{a}_{1}, b_{1}\right), \ldots,\left(\vec{a}_{l}, b_{l}\right)$ are the generators of the cone $\operatorname{proj}_{\mathbf{x}}(\mathcal{R})^{\#}$, then $\mathcal{R}_{k+1}=\mathcal{R}_{k} \wedge-\vec{a}_{1} \cdot \mathbf{y} \leq 0 \wedge \cdots \wedge-\vec{a}_{l} \cdot \mathbf{y} \leq 0$.

Proof. Follows from the fact that $\operatorname{proj}_{\mathbf{x}}\left(\mathcal{Q}_{k}\right)=\operatorname{proj}_{\mathbf{x}}\left(\mathcal{R}_{k}\right)$, and thus $\operatorname{proj}_{\mathbf{x}}\left(\mathcal{Q}_{k}\right)^{\#}$ and $\operatorname{proj}_{\mathbf{x}}\left(\mathcal{R}_{k}\right)^{\#}$ are the same, and that for $\rho(\mathbf{x})=\vec{a} \cdot \mathbf{x}+b$ we have $\Delta \rho\left(\mathbf{x}^{\prime \prime}\right)=\rho(\mathbf{x})-\rho\left(\mathbf{x}^{\prime}\right)=$ $-\vec{a} \cdot \mathbf{y}$, by definition of the displacement polyhedron.

LEMMA 5.3. Let $\left(\vec{a}_{1}, b_{1}\right), \ldots,\left(\vec{a}_{l}, b_{l}\right)$ be the generators of the cone $\operatorname{proj}_{\mathbf{x}}(\mathcal{R})^{\#}$, then $-\vec{a}_{1} \cdot \mathbf{y} \leq 0 \wedge \cdots \wedge-\vec{a}_{l} \cdot \mathbf{y} \leq 0$ of Lemma 5.2 is equivalent to $M \mathbf{y} \leq 0$, where $M$ is such that $\operatorname{proj}_{\mathbf{x}}(\mathcal{R}) \equiv[M \mathbf{x} \leq \mathbf{b}]$.

Proof. Consider $(\vec{a}, b) \in \operatorname{proj}_{\mathbf{x}}(\mathcal{Q})^{\#}=\operatorname{proj}_{\mathbf{x}}(\mathcal{R})^{\#}$. By Farkas' lemma, a function $f(\mathbf{x})=\vec{a} \cdot \mathbf{x}+b$ is nonnegative over $\operatorname{proj}_{\mathbf{x}}(\mathcal{R})$ iff there are nonnegative $\vec{\lambda}=\left(\lambda_{1}, \ldots, \lambda_{m}\right)$ such that $\vec{\lambda} \cdot M=-\vec{a} \wedge \vec{\lambda} \cdot \mathbf{b} \leq b$. Note that any (nonnegative) values for $\vec{\lambda}$ define corresponding values for $\vec{a}$ and $b$. Thus the valid values for $\vec{a}$ are all conic combinations of the rows of $-M$, i.e., this cone is generated by the rows of $-M$. Hence $-\vec{a}_{1} \cdot \mathbf{y} \leq$ $0 \wedge \cdots \wedge-\vec{a}_{l} \cdot \mathbf{y} \leq 0$ is equivalent to $M \mathbf{y} \leq \mathbf{0}$.

We use the above lemma to show that $\mathcal{R}_{k}$ can be represented as in (35), without the need to compute $M$ explicitly. We first note that using lemmas 5.2 and 5.3 we get that $\mathcal{R}_{k+1}=\mathcal{R}_{k} \cap \mathcal{D}_{k}$, where

$$
\begin{aligned}
\mathcal{D}_{k} & =\left\{\left(\begin{array}{l}
\mathbf{x} \\
\mathbf{y}
\end{array}\right) \in \mathbb{Q}^{2 n} \mid M \mathbf{y} \leq \mathbf{0}\right\} \quad(M \text { as in Lemma 5.3) }) \\
& =\left\{\left(\begin{array}{l}
\mathbf{x} \\
\mathbf{y}
\end{array}\right) \in \mathbb{Q}^{2 n} \mid \mathbf{y} \in \text { rec.cone }\left(\operatorname{proj}_{\mathbf{x}}\left(\mathcal{R}_{k}\right)\right)\right\} .
\end{aligned}
$$

LEMMA 5.4. $\mathcal{R}_{k}=\operatorname{proj}_{\mathbf{x}, \mathbf{y}_{0}}\left(\mathcal{R}_{k}^{\prime}\right)$ where $\mathcal{R}_{k}^{\prime}$ is defined by (35).

Proof. We use induction on $k$. For $k=0$ the lemma states that $\mathcal{R}_{0}$ is equal $R\left(\begin{array}{l}\mathbf{x} \\ \mathbf{y}_{0}\end{array}\right) \leq \mathbf{c}^{\prime \prime}$, which is correct since by definition $\mathcal{R}_{0}=\mathcal{R}$. Assume the lemma holds for $\mathcal{R}_{k}$, we prove it for $\mathcal{R}_{k+1}=\mathcal{R}_{k} \cap \mathcal{D}_{k}$. By the induction hypothesis,

$$
\mathcal{R}_{k}=\left\{\left(\begin{array}{l}
\mathbf{x} \\
\mathbf{y}_{0}
\end{array}\right) \in \mathbb{Q}^{2 n} \mid R\left(\begin{array}{l}
\mathbf{x} \\
\mathbf{y}_{0}
\end{array}\right) \leq \mathbf{c}^{\prime \prime} \wedge R\left(\begin{array}{l}
\mathbf{y}_{0} \\
\mathbf{y}_{1}
\end{array}\right) \leq \mathbf{0} \wedge \ldots \wedge R\left(\begin{array}{l}
\mathbf{y}_{k-1} \\
\mathbf{y}_{k}
\end{array}\right) \leq \mathbf{0}\right\}
$$


and

$$
\begin{array}{rlr}
\mathcal{D}_{k} & =\left\{\left(\begin{array}{l}
\mathbf{x} \\
\mathbf{y}_{0}
\end{array}\right) \in \mathbb{Q}^{2 n} \mid \mathbf{y}_{0} \in \operatorname{rec} . \operatorname{cone}\left(\operatorname{proj}_{\mathbf{x}}\left(\mathcal{R}_{k}\right)\right)\right\} & \text { by definition } \\
& =\left\{\left(\begin{array}{l}
\mathbf{x} \\
\mathbf{y}_{0}
\end{array}\right) \in \mathbb{Q}^{2 n} \mid \mathbf{y}_{0} \in \operatorname{rec} . \operatorname{cone}\left(\operatorname{proj}_{\mathbf{x}}\left(\operatorname{proj}_{\mathbf{x}, \mathbf{y}_{0}}\left(\mathcal{R}_{k}^{\prime}\right)\right)\right)\right\} & \\
& =\left\{\left(\begin{array}{l}
\mathbf{x} \\
\mathbf{y}_{0}
\end{array}\right) \in \mathbb{Q}^{2 n} \mid \mathbf{y}_{0} \in \operatorname{rec} . \operatorname{cone}\left(\operatorname{proj}_{\mathbf{x}}\left(\mathcal{R}_{k}^{\prime}\right)\right)\right\} & \\
& =\left\{\left(\begin{array}{l}
\mathbf{x} \\
\mathbf{y}_{0}
\end{array}\right) \in \mathbb{Q}^{2 n} \mid \mathbf{y}_{0} \in \operatorname{proj}_{\mathbf{x}}\left(\operatorname{rec} \cdot \operatorname{cone}\left(\mathcal{R}_{k}^{\prime}\right)\right)\right\} & \text { by Lemma } 2.1 \\
& =\left\{\left(\begin{array}{l}
\mathbf{x} \\
\mathbf{y}_{0}
\end{array}\right) \in \mathbb{Q}^{2 n} \mid R\left(\begin{array}{l}
\mathbf{y}_{0} \\
\mathbf{y}_{1}
\end{array}\right) \leq \mathbf{0} \wedge R\left(\mathbf{y}_{1}\right) \leq \mathbf{0} \wedge \cdots \wedge R\left(\begin{array}{l}
\mathbf{y}_{k} \\
\mathbf{y}_{k+1}
\end{array}\right) \leq \mathbf{0}\right\}
\end{array}
$$

Note that in the last step, we incorporated the recession cone of $\mathcal{R}_{k}^{\prime}$ as in (35), after renaming $\mathbf{y}_{i}$ to $\mathbf{y}_{i+1}$, and $\mathbf{x}$ to $\mathbf{y}_{0}$ just to make it easier to read in the next step. Now, let us compute $\mathcal{R}_{k+1}=\mathcal{R}_{k} \cap \mathcal{D}_{k}$. Note that any $\left(\begin{array}{l}\mathbf{x} \\ \mathbf{y}_{0}\end{array}\right) \in \mathcal{R}_{k+1}$ must satisfy the constraint $R\left(\begin{array}{c}\mathbf{y}_{0} \\ \mathbf{y}_{0}\end{array} \leq \mathbf{c}^{\prime \prime}\right.$ that comes form $\mathcal{R}_{k}$. Adding this constraint to $\mathcal{D}_{k}$ above we clearly obtain a subset of $\mathcal{R}_{k}$, and thus

$$
\mathcal{R}_{k+1}=\left\{\left(\begin{array}{l}
\mathbf{x} \\
\mathbf{y}_{0}
\end{array}\right) \mid R\left(\begin{array}{l}
\mathbf{x} \\
\mathbf{y}_{0}
\end{array}\right) \leq \mathbf{c}^{\prime \prime} \wedge R\left(\begin{array}{l}
\mathbf{y}_{0} \\
\mathbf{y}_{1}
\end{array}\right) \leq \mathbf{0} \wedge \cdots \wedge R\left(\begin{array}{l}
\mathbf{y}_{k-1} \\
\mathbf{y}_{k}
\end{array}\right) \leq \mathbf{0}\right\}
$$

which is exactly $\operatorname{proj}_{\mathbf{x}, \mathbf{y}_{0}}\left(\mathcal{R}_{k+1}^{\prime}\right)$, justifying the lemma's statement for $k+1$.

LEMMA 5.5. $\mathcal{Q}$ has a $M \Phi R F$ of depth d iff $\mathcal{R}_{d}^{\prime}$ is empty.

Proof. By Lemma 3.6 $\mathcal{Q}$ has a M MRF of depth $d$ iff $\mathcal{Q}_{d}=F^{d}(\mathcal{Q})$ is empty, and by Definition 5.1 $\mathcal{Q}_{d}$ is empty iff $\mathcal{R}_{d}$ is empty, and since $\mathcal{R}_{d}$ is empty iff $\mathcal{R}_{d}^{\prime}$ is empty the lemma follows.

Apart from providing a polynomial-time implementation for the check that $F^{d}(\mathcal{Q})=$ $\emptyset$, we believe that the above lemma provides us with a new tool for addressing the problem of deciding whether a given $S L C$ loop has a $M \Phi R F$ of any depth, which is still an open problem. Next we discuss some directions.

One direction is to come up with conditions on the matrices $A^{\prime \prime}$ (or equivalently $R$ ) and $\mathbf{c}^{\prime \prime}$ under which it is guaranteed that if $\mathcal{R}_{k}^{\prime}$ is empty then $k$ must be smaller than some $d$, i.e., bounding the depth of M $\Phi$ RFs for classes of loops that satisfy these conditions. We can also view the problem as looking for some $N$, such that $\mathcal{C}^{N}=\mathcal{C}^{N+1}$ where $\mathcal{C}=\left[R\left(\begin{array}{c}\mathbf{y} \\ \mathbf{y}^{\prime}\end{array}\right) \leq \mathbf{0}\right]$, which is a sufficient condition for the algorithm to terminate in at most $N$ iterations, since then $\mathcal{R}_{N}=\mathcal{R}_{N+1}$, either with a recurrent set or with a M $\Phi$ R. This is particularly interesting if the loop is deterministic with affine update as in (5). In such case $\mathcal{C}=\left[B \mathbf{y} \leq 0 \wedge \mathbf{y}^{\prime}=(U-I) \mathbf{y}\right]$, where $I \in \mathbb{Q}^{n \times n}$ is the identity matrix, and thus if the matrix $(U-I)$ is nilpotent, for example, then there is $N$ such that $\mathcal{C}^{N}=\mathcal{C}^{N+1}$. This also holds when matrix $(U-I)$ satisfies the finite-monoid property discussed in Section 4.3 . 
Another tantalizing observation reduces the existence of $d$ such that $\mathcal{R}_{d}^{\prime}$ is empty to the question whether a related $S L C$ loop terminates, for a given polyhedron of initial states, in a bounded number of steps. Specifically, the loop:

$$
\text { while }(B \mathbf{y} \leq \mathbf{0}) \text { do }\left(A+A^{\prime}\right) \mathbf{y}+A^{\prime} \mathbf{y}^{\prime} \leq \mathbf{0} \text {. }
$$

where $B, A$ and $A^{\prime}$ are those used in the definition of $R$, and the question whether it terminates in at most $d$ steps for all $\mathbf{y} \in\left\{\mathbf{y} \in \mathbb{Q}^{n} \mid R\left(\begin{array}{l}\mathbf{x} \\ \mathbf{y}\end{array}\right) \leq \mathbf{c}^{\prime \prime}\right\}$. Note that if the update is affine as in (5), then the above loop is equivalent to

$$
\text { while }(B \mathbf{y} \leq \mathbf{0}) \text { do } \mathbf{y}^{\prime}=(U-I) \mathbf{y}
$$

where $I \in \mathbb{Q}^{n \times n}$ is the identity matrix.

To further demonstrate the usefulness of the displacement polyhedra, next we provide some observations, regarding $S L C$ loops with bounded transition polyhedra, that are easy to see using the displacement polyhedron and are much less obvious using the transition polyhedron. Recall that a transition polyhedron is bounded if its recession cone consists of a single point $(\mathbf{0}, \mathbf{0})$.

LEMMA 5.6. A bounded $S L C$ loop $\mathcal{Q}$ it is nonterminating iff it has a fixpoint $(\mathbf{x}, \mathbf{x}) \in$ $\mathcal{Q}$, and it is terminating iff it has a $L R F$.

Proof. Since $\mathcal{Q}$ is bounded, the displacement polyhedron $\mathcal{R} \equiv\left[R\left(\begin{array}{l}\mathbf{x} \\ \mathbf{y}\end{array}\right) \leq \mathbf{c}^{\prime \prime}\right]$ is bounded, and its recession cone $R\left(\begin{array}{l}\mathbf{x} \\ \mathbf{y}\end{array}\right) \leq \mathbf{0}$ consists of a single point $(\mathbf{0}, \mathbf{0})$. From the form of $\mathcal{R}_{k}^{\prime}$, which is a conjunction of instances of $R\left(\mathbf{y}_{i+1}\right) \leq \mathbf{0}$, it is easy to see that $\mathcal{R}_{2}=\mathcal{R}_{1}$. This means that the algorithm will terminate in at most two iterations with one of the following outcomes: (i) $\mathcal{R}_{0}=\mathcal{R}_{1}$; (ii) $\mathcal{R}_{2}=\mathcal{R}_{1}$; or (iii) $\mathcal{R}_{1}$ is empty. In the first two cases all transitions of $\mathcal{R}_{1}$ or $\mathcal{R}_{2}$ are of the form $\left(\begin{array}{l}\mathbf{x} \\ \mathbf{0}\end{array}\right)$, and thus $(\mathbf{x}, \mathbf{x}) \in \mathcal{Q}$; in the third case we have found a $M \Phi R F$ of depth 1, i.e., $L R F$. Note that the part that relates nontermination to the existence of a fixpoint follows also from [27] as well.

\section{Conclusions}

The purpose of this work has been to improve our understanding of M $\Phi$ RFs, in particular of the problem of deciding whether a given $S L C$ loop has a M $\Phi$ F without a given bound on the depth. The outcomes are important insights that shed light on the structure of these ranking functions.

At the heart of our work is an algorithm that seeks M $\Phi$ RFs, which is based on iteratively eliminating transitions, that satisfy some property, until eliminating them 
all or stabilizing on a set of transitions that cannot be reduced anymore. In the first case, a M $\Phi R F$ can be constructed, and, surprisingly, in the second case the stable set of transitions turned to be a recurrent set that witnesses nontermination. This reveals an equivalence between the problems of seeking M $\Phi R F$ s and seeking recurrent sets of a particular form.

Apart from the relation to seeking recurrent sets, the insights of our work are helpful for characterizing classes of loops for which there is a always a M $\Phi R F$, when terminating. We demonstrated this for two classes that have been considered previously. In addition, our insights led to a new representation for $S L C$ in which our algorithm has a very simple formalization, and, moreover, it allows making nontrivial observations regards (bounded) $S L C$ loop straightforward. We believe that this representation can be useful for other related problems. Our research leaves as well a considerable amount of related new open questions, which we hope will trigger the interest of the community in this line of work.

An implementation of Algorithm 1 is available at http://www . loopkiller.com/irankf inder - options $M \Phi R F(\mathbb{Q})$ or $M \Phi R F(\mathbb{Z})$ - together with corresponding examples of $S L C$ loops. For experimentally evaluating Algorithm 1 for nontermination, we have integrated it in a newer version of iRANKFINDER that takes as input a control-flow graph, as follows: when it fails to prove termination, it enumerates closed walks (which are basically $S L C$ loops) using only transitions whose termination was not proven, and then applies Algorithm 1 to seek recurrent sets. For now it does not check that the recurrent set is reachable, which is an orthogonal problem. We have analyzed 439 benchmarks taken from TPDB [1] for which iRANKFInDER fails to prove termination For 400 it find recurrent sets, out of which 273 include a fixpoint transition. For the remaining 127 benchmarks, 114 are defined by octagonal relations and 114 are deterministic. All are available at http://irankfinder.loopkiller.com, under the folder Papers/mlrf_recset, and can be analyzed by enabling Nontermination in the settings section.

The problem of seeking M $\Phi R F$ s with a given bound on the depth has been considered before in several works. The complexity and algorithmic aspects has been completely settled in [8]. M $\Phi$ RFs for general loops have been considered in [26, 29], where both use non-linear constraint solving. In [4] the notion of "eventual linear ranking functions," which are M $\Phi R F$ s of depth 2 has been studied. The approach described in [9] is also able to infer M $\Phi R F$ s for general loops incrementally, by solving corresponding safety problems using Max-SMT. Lexicographic ranking function are very related, their algorithmic and complexity aspects has been considered in several works [11, 3, 7, 25].

Nontermination of programs or corresponding control-flow graphs has been considered before in several works: some [22, 24, 31, 6, 5, 14, 10, 27] are based on finding 
recurrent sets in one form or another; while others are based on reducing the problem to proving non-reachability of terminating states [15, 36].

\section{References}

[1] The Termination Problems Data Base. http://termination-portal.org/wiki/TPDB.

[2] Elvira Albert, Puri Arenas, Samir Genaim, and Germán Puebla. Closed-form upper bounds in static cost analysis. J. Autom. Reasoning, 46(2):161-203, 2011.

[3] Christophe Alias, Alain Darte, Paul Feautrier, and Laure Gonnord. Multidimensional rankings, program termination, and complexity bounds of flowchart programs. In Radhia Cousot and Matthieu Martel, editors, Static Analysis Symposium, SAS'10, volume 6337 of LNCS, pages 117-133. Springer, 2010.

[4] Roberto Bagnara and Fred Mesnard. Eventual linear ranking functions. In Proceedings of the 15th International Symposium on Principles and Practice of Declarative Programming, PPDP 2013, pages 229-238. ACM Press, 2013.

[5] Alexey Bakhirkin, Josh Berdine, and Nir Piterman. A forward analysis for recurrent sets. In Sandrine Blazy and Thomas Jensen, editors, Static Analysis Symposium, SAS'15, volume 9291 of Lecture Notes in Computer Science, pages 293-311. Springer, 2015.

[6] Alexey Bakhirkin and Nir Piterman. Finding recurrent sets with backward analysis and trace partitioning. In Marsha Chechik and Jean-François Raskin, editors, Tools and Algorithms for the Construction and Analysis of Systems, TACAS'16, volume 9636 of Lecture Notes in Computer Science, pages 17-35. Springer, 2016.

[7] Amir M. Ben-Amram and Samir Genaim. Ranking functions for linear-constraint loops. Journal of the ACM, 61(4):26:1-26:55, jul 2014.

[8] Amir M. Ben-Amram and Samir Genaim. On multiphase-linear ranking functions. In Rupak Majumdar and Viktor Kuncak, editors, Computer Aided Verification, CAV'17, volume 10427 of Lecture Notes in Computer Science, pages 601-620. Springer, 2017.

[9] Cristina Borralleras, Marc Brockschmidt, Daniel Larraz, Albert Oliveras, Enric Rodríguez-Carbonell, and Albert Rubio. Proving termination through conditional termination. In Axel Legay and Tiziana Margaria, editors, Tools and Algorithms 
for the Construction and Analysis of Systems, TACAS'17, volume 10205 of Lecture Notes in Computer Science, pages 99-117, 2017.

[10] Marius Bozga, Radu Iosif, and Filip Konecný. Deciding conditional termination. Logical Methods of Computer Science, 10(3), 2014.

[11] Aaron R. Bradley, Zohar Manna, and Henny B. Sipma. Linear ranking with reachability. In Kousha Etessami and Sriram K. Rajamani, editors, Computer Aided Verification, CAV'05, volume 3576 of LNCS, pages 491-504. Springer, 2005.

[12] Mark Braverman. Termination of integer linear programs. In Thomas Ball and Robert B. Jones, editors, Computer Aided Verification, CAV'06, volume 4144 of LNCS, pages 372-385. Springer, 2006.

[13] Marc Brockschmidt, Fabian Emmes, Stephan Falke, Carsten Fuhs, and Jürgen Giesl. Analyzing runtime and size complexity of integer programs. ACM Trans. Program. Lang. Syst., 38(4):13, 2016.

[14] Marc Brockschmidt, Thomas Ströder, Carsten Otto, and Jürgen Giesl. Automated detection of non-termination and nullpointerexceptions for java bytecode. In Bernhard Beckert, Ferruccio Damiani, and Dilian Gurov, editors, Formal Verification of Object-Oriented Software, FoVeOOS'11, volume 7421 of Lecture Notes in Computer Science, pages 123-141. Springer, 2011.

[15] Hong Yi Chen, Byron Cook, Carsten Fuhs, Kaustubh Nimkar, and Peter W. O'Hearn. Proving nontermination via safety. In Erika Ábrahám and Klaus Havelund, editors, Tools and Algorithms for the Construction and Analysis of Systems, TACAS'14, volume 8413 of Lecture Notes in Computer Science, pages 156-171. Springer, 2014.

[16] Michael Colón and Henny Sipma. Synthesis of linear ranking functions. In Tiziana Margaria and Wang Yi, editors, Tools and Algorithms for the Construction and Analysis of Systems, TACAS'01, volume 2031 of LNCS, pages 67-81. Springer, 2001.

[17] Michele Conforti, Gérard Cornuéjols, and Giacomo Zambelli. Polyhedral approaches to mixed integer linear programming. In Jünger et al., editor, 50 Years of Integer Programming 1958-2008, pages 343-386. Springer, 2010. 
[18] Byron Cook, Alexey Gotsman, Andreas Podelski, Andrey Rybalchenko, and Moshe Y. Vardi. Proving that programs eventually do something good. In Proceedings of the 34th ACM SIGPLAN-SIGACT Symposium on Principles of Programming Languages, POPL 2007, Nice, France, January 17-19, 2007, pages 265-276, 2007.

[19] Byron Cook, Sumit Gulwani, Tal Lev-Ami, Andrey Rybalchenko, and Mooly Sagiv. Proving conditional termination. In Computer Aided Verification, CAV'08, volume 5123 of Lecture Notes in Computer Science, pages 328-340. Springer, 2008.

[20] Byron Cook, Andreas Podelski, and Andrey Rybalchenko. Termination proofs for systems code. In Michael I. Schwartzbach and Thomas Ball, editors, Programming Language Design and Implementation, PLDI'06, pages 415-426. ACM, 2006.

[21] Paul Feautrier. Some efficient solutions to the affine scheduling problem. I. Onedimensional time. International Journal of Parallel Programming, 21(5):313-347, 1992.

[22] Ashutosh Gupta, Thomas A. Henzinger, Rupak Majumdar, Andrey Rybalchenko, and Ru-Gang Xu. Proving non-termination. In George C. Necula and Philip Wadler, editors, Symposium on Principles of Programming Languages, POPL'08, pages 147-158, 2008.

[23] Michael Harrison. Lectures on Sequential Machines. Academic Press, 1969.

[24] Daniel Larraz, Kaustubh Nimkar, Albert Oliveras, Enric Rodríguez-Carbonell, and Albert Rubio. Proving non-termination using max-smt. In Armin Biere and Roderick Bloem, editors, Computer Aided Verification, CAV'14, volume 8559 of Lecture Notes in Computer Science, pages 779-796. Springer, 2014.

[25] Daniel Larraz, Albert Oliveras, Enric Rodríguez-Carbonell, and Albert Rubio. Proving termination of imperative programs using Max-SMT. In Formal Methods in Computer-Aided Design, FMCAD 2013, pages 218-225. IEEE, 2013.

[26] Jan Leike and Matthias Heizmann. Ranking templates for linear loops. Logical Methods in Computer Science, 11(1):1-27, 2015.

[27] Jan Leike and Matthias Heizmann. Geometric nontermination arguments. In Dirk Beyer and Marieke Huisman, editors, Tools and Algorithms for the Construction and Analysis of Systems, TACAS'18, volume 10806 of Lecture Notes in Computer Science, pages 266-283. Springer, 2018. 
[28] Jérôme Leroux and Grégoire Sutre. Flat counter automata almost everywhere! In International Symposium on Automated Technology for Verification and Analysis (ATVA 2005), volume 3707 of Lecture Notes in Computer Science, pages 489-503. Springer, 2005.

[29] Yi Li, Guang Zhu, and Yong Feng. The L-depth eventual linear ranking functions for single-path linear constraint loops. In 10th International Symposium on Theoretical Aspects of Software Engineering (TASE 2016), pages 30-37. IEEE, 2016.

[30] Joël Ouaknine and James Worrell. On linear recurrence sequences and loop termination. ACM SIGLOG News, 2(2):4-13, April 2015.

[31] Étienne Payet, Fred Mesnard, and Fausto Spoto. Non-termination analysis of java bytecode. CoRR, abs/1401.5292, 2014.

[32] Andreas Podelski and Andrey Rybalchenko. A complete method for the synthesis of linear ranking functions. In Bernhard Steffen and Giorgio Levi, editors, Verification, Model Checking, and Abstract Interpretation, VMCAI'04, volume 2937 of LNCS, pages 239-251. Springer, 2004.

[33] Alexander Schrijver. Theory of Linear and Integer Programming. John Wiley and Sons, New York, 1986.

[34] Kirack Sohn and Allen Van Gelder. Termination detection in logic programs using argument sizes. In Daniel J. Rosenkrantz, editor, Symposium on Principles of Database Systems, pages 216-226. ACM Press, 1991.

[35] Ashish Tiwari. Termination of linear programs. In Rajeev Alur and Doron Peled, editors, Computer Aided Verification, CAV'04, volume 3114 of LNCS, pages 387390. Springer, 2004.

[36] Helga Velroyen and Philipp Rümmer. Non-termination checking for imperative programs. In Bernhard Beckert and Reiner Hähnle, editors, Tests and Proofs, TAP'08, volume 4966 of Lecture Notes in Computer Science, pages 154-170. Springer, 2008. 